\title{
11. LATE QUATERNARY POLYCYSTINE RADIOLARIANS AND SILICOFLAGELLATES OF A DIATOMACEOUS SAPROPEL FROM THE EASTERN MEDITERRANEAN, SITES 969 AND 971
}

\author{
Taniel Danelian ${ }^{2}$ and Dimitris Frydas ${ }^{3}$
}

\begin{abstract}
Well-preserved radiolarians and silicoflagellates discovered in the diatomaceous Sapropel S5 were studied and quantitatively analyzed. The silicoflagellate assemblage is almost exclusively composed of warm-water Dictyocha species. The radiolarian assemblage is very diverse and dominated by the counting-group Arachnocorallium, an indicator of eutrophication. Paleoecological evaluation of the fauna indicates that polycystin radiolarians occupied various ecological niches. Radiolarian taxa, known to be abundant in association with a subsurface layer of chlorophyll- $\alpha$ maxima are much better represented in the sapropel S5 than above it, suggesting that this deep layer of primary productivity was much stronger and better developed during accumulation of the sapropel. Deep-dwelling, detritivore radiolarians are also present and well-represented in the sapropel, contrary to the assemblage that is above it, in which these taxa appear to be absent. In the latter sample, surface-dwelling, symbiont-bearing radiolarians are the dominant group.

If the Nile river was the main cause of eutrophication in the Eastern Mediterranean, excessive riverine discharge would have supplied abundant nutrients (in particular, phosphorus and silica) to sustain the bloom of the siliceous phytoplankton (diatoms and silicoflagellates). Eutrophication would have encouraged radiolarians to occupy ecological niches at various depths of the water column. Excessive export of biogenic silica (diatom shells in particular) diminished the corrosive action of the Mediterranean sea water and preserved siliceous microfossils. After the decrease of riverine discharge, which brought an end to the formation of Sapropel S5, silica was probably not available in sufficient quantities to sustain siliceous phytoplankton growth far beyond the coasts of Egypt, and settling of organic matter would have been reduced to levels below the critical values necessary for the sustenance of the detritivore radiolarian niches. This change in eutrophication did not affect the surface-dwelling radiolarians, whose needs are substantially supported by their symbionts.
\end{abstract}

\section{INTRODUCTION}

The occurrence of siliceous microfossils, such as radiolarians (zooplankton), silicoflagellates and diatoms (phytoplankton), is generally very scarce in the Pliocene-Quaternary sediments of the Eastern Mediterranean, which is largely dominated by carbonate-rich sediments. However, opal assemblages are exceptionally well-preserved in some organic-rich levels, known as sapropels (Dumitrica, 1973a, 1973b; Schrader and Matherne, 1981; Riedel et al., 1985; Anastasakis and Filippas, 1990). High accumulation rates of biogenic silica are regarded as a good fossil indicator of eutrophication (Brasier, 1995) and there is a general association in the geological record of organic carbon-rich sediments and abundant biogenic silica. Having been the main siliceous planktonic group for most of pre-Cenozoic times, radiolarians are often associated with hydrocarbon source rocks and, given the presence of lipids in their living representatives (Anderson, 1983), they themselves may well have been significant contributors to the accumulation of organic-rich sediments (Ormiston, 1993).

In order to understand the paleoceanographic and depositional processes that led to the accumulation and preservation of sapropels in the Eastern Mediterranean (a main topic of the ODP Leg 160; Emeis and Shipboard Scientific Party, 1996) scientific interest has focused on their age and cyclicity, sedimentology, mineralogy and geochemistry and their fauna/flora. The latter are the subject of the present paper. Sapropel accumulation in the Eastern Mediterranean sediments has the character of a synchronous event and it is widely

${ }^{1}$ Robertson, A.H.F., Emeis, K.-C., Richter, C., and Camerlenghi, A. (Eds.), 1998. Proc. ODP, Sci. Results, 160: College Station, TX (Ocean Drilling Program).

${ }^{2}$ Department of Geology and Geophysics, The University of Edinburgh, West Mains Road, Edinburgh EH9 3JW, United Kingdom. Taniel.Danelian@ed.ac.uk

${ }^{3}$ Patras University, Department of Geology, Section for Physical-Marine Geology and Geodynamics, GR-26110, Patras, Greece. regarded as the result of climate-driven external forcing. More particularly, a temporal correlation has been observed between the accumulation of sapropels and the precessional cycle of earth's orbit. Furthermore, a link has been established between the sapropels and (1) intensifications of the African monsoons, which affected the discharge of the Nile river (Rossignol-Strick, 1983) and (2) increased activity of Mediterranean depressions followed by enhanced humidity in the northern borderlands of the Eastern Mediterranean (Rohling, 1994). Geochemical evidence indicates that sapropel formation has been promoted by increased productivity (Calvert et al., 1992; Howell and Thunell, 1992). However, agreement has not yet been reached concerning the mechanism(s) that conveyed sapropel accumulation, and more especially their preservation, onto the Mediterranean seafloor (Pedersen and Calvert, 1990).

In recent years paleoecological information from the fauna and flora included in the Pliocene-Quaternary sediments has provided new insights to the puzzling question of sapropel formation in the Eastern Mediterranean, a basin reputed to be "a nutrient desert in a nutrient desert" (Emeis and Shipboard Scientific Party, 1996). More particularly, paleoecological analysis of planktonic foraminifers (Rohling and Gieskes, 1989) and calcareous nannofossils (Castradori, 1993) from the sapropels has brought forward new and elaborated models concerning the depths and intensity of primary paleoproductivity. Contrary to calcareous plankton, the siliceous plankton preserved in the sapropels have not yet been well-explored, mainly because of their sparsity in the sedimentary record.

Among the many sapropel layers discovered to date in the Pliocene-Quaternary sediments of the Eastern Mediterranean, the Late Pleistocene Sapropel S5 is of particular importance. Abundant and well-preserved opal assemblages were studied by Schrader and Matherne (1981), although these authors focused their study on diatoms and reported only a few silicoflagellates. The abundant biogenic silica preserved in the Sapropel S5 seems to cover a significantly extensive area of the Eastern Mediterranean south of Crete (Fig. 1), and 
during ODP Leg 160, well-preserved siliceous assemblages from this sapropel were encountered at Site 971 (Shipboard Scientific Party, 1996b, p. 435). The radiolarian and silicoflagellate assemblages from this level are discussed and extensively documented here for the first time. Moreover, radiolarians discovered a few centimeters above the Sapropel S5 at Site 969 provide a good opportunity to observe the long-term changes incurred by radiolarian populations during and just after the accumulation of the Sapropel S5.

\section{MATERIAL AND METHODS}

Sites 969 and 971 are close to each other and are both situated at depths of 2100-2200 m on the Mediterranean Ridge, south of Crete (Fig. 1). No radiolarian or silicoflagellate specialist was included in the shipboard party of Leg 160; therefore, this investigation is based on samples selected on the ship by other specialists. Radiolarians were studied by T. Danelian and silicoflagellates by D. Frydas. Sample 160-971B-2H-CC comes from the 30-cm-thick diatom-rich sapropel S5, which was drilled in the center of a moat situated at the southeastern edge of the Napoli mud volcano (Robertson et al., 1996). In this sample, radiolarians are very well preserved and diverse. The studied sample is of late Pleistocene age (younger than $0.26 \mathrm{Ma}$ ) and is situated between Subzone MNN 21a and Zone MNN 20 of the calcareous nannofossils (Shipboard Scientific Party, 1996b). More precisely, Sapropel S5 accumulated during the warm isotopic stage 5e, at approximately $125 \mathrm{Ka}$ (Rossignol-Strick, 1983). Sample 160-969A-1H-3, 65-67 cm, comes from a level situated a few centimeters above the same sapropel (S5) and is of late Pleistocene age too (Zone MNN21, Shipboard Scientific Party, 1996a). At the time of receipt this sample was already washed and sieved and the residue contained radiolarians of moderate preservation, but no silicoflagellates were encountered in it. Sample 160-971B-2H-CC was washed under a $45-\mu \mathrm{m}$ sieve and slides were prepared according to Goll and Bjørklund (1989).

\section{RADIOLARIAN RESULTS AND SYSTEMATICS}

Radiolarians from the Mediterranean plankton and sediments were the subject of the very first classical works on this group (Müller, 1858; Ehrenberg, 1858; Haeckel, 1860a, 1860b). However, despite this early interest and substantial efforts during the seventies

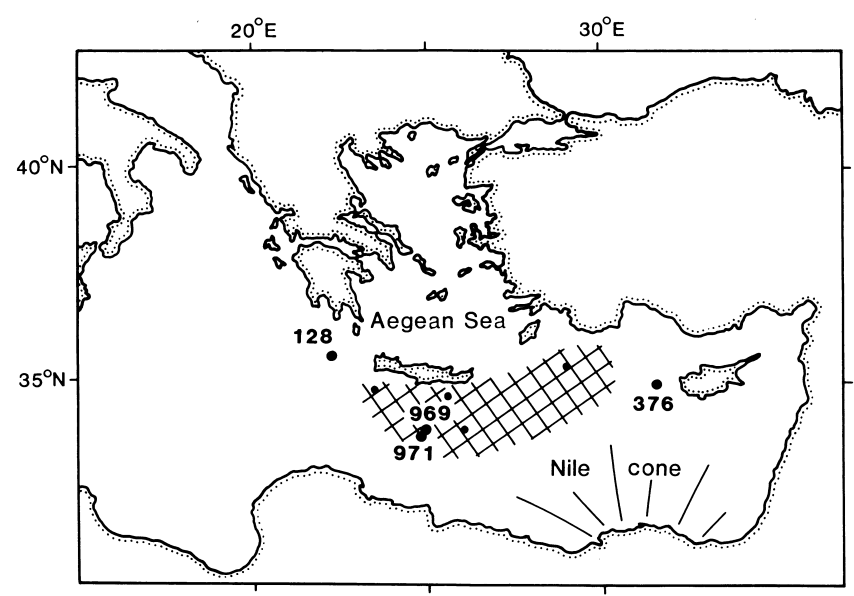

Figure 1. Eastern Mediterranean DSDP Sites 128 and 376 and ODP Sites 969 and 971 with Quaternary radiolaria. The hatched area indicates the inferred distribution of siliceous microfossil occurrence in sapropel S5 (after Schrader and Matherne, 1981). Small black circles without site numbers indicate study cores in which the above authors found diatoms. to document the Late Cenozoic radiolarian assemblages of the Mediterranean (Sanfilippo, 1971; Dumitrica, 1973a; Sanfilippo et al., 1973; Caulet, 1974; Riedel et al., 1974; Sanfilippo and Riedel, 1975) our knowledge is still far from complete. This is especially true for the Pliocene-Quaternary sediments, in which radiolarians are very sparse, but their presence is even more important, because it may have had a particular paleoenvironmental/paleoceanographic significance (e.g., increased biological productivity; Riedel et al., 1985). Despite the rarity of radiolarian assemblages in the sedimentary record, differences in their structure at family level were noticed and appeared to provide ground for paleoecological interpretations (Sanfilippo, 1971). Riedel et al. (1985) examined quantitatively a number of late Neogene radiolarian samples from the Eastern Mediterranean with a paleoenvironmental perspective in mind. Two of their samples are of Quaternary age and come from the west of Crete (128-3-5) and Cyprus (376-1-4; see Fig. 1). These authors successfully introduced the "counting-group" approach, at the approximate level of genera, to describe the composition of their studied radiolarian assemblages.

To explore the paleoenvironmental significance of the radiolarian assemblages from samples from ODP Leg 160, the approach of Riedel et al. (1985) was adopted. We therefore quantified the composition of radiolarian assemblages on the basis of $~ 500$ specimens encountered on traverses of strewn-slide preparations. The numbers in Table 1 refer to the taxa studied by Riedel et al. (1985, see their table 23.1 for comparison).

\section{Radiolarian Systematics}

For each taxon mentioned herein there is at least one reference in the synonymy list that contains appropriate descriptions and illustrations. As data on Quaternary Radiolaria from the Eastern Mediterranean are sparse, this list links observations of the present study with previous findings. In order to investigate dominant counting-groups in our material (indicated in bold), the taxonomic scheme of Riedel et al. (1985) was followed in this study and, therefore, only a few taxa are dealt with at species level. In general, each counting-group is identified as "spp." or "group." The former was used when the above authors had reason to believe that there was more than one species present, and "group" when they did not know whether there was more than one species (W. Riedel, pers. comm., 1996).

\section{Subclass RADIOLARIA \\ Superorder POLYCYSTINA \\ Order SPUMELLARIA}

Family COLLOSPHAERIDAE Müller, 1858

Collosphaerid group

(Pl. 2, Figs. 1-2)

Collosphaerid group; Riedel et al., 1985, p. 504, pl.1, fig.1a-d.

Acrosphaera spinosa (Haeckel) group?

(Pl. 2, Fig. 1)

Polysolenia spinosa (Haeckel); Dumitrica, 1973a, p. 832, pl. 18, fig. 2. Acrosphaera spinosa (Haeckel); Van de Paverd, 1995, p. 49, pl. 5-7.

Acrosphaera spinosa (Haeckel) group?; Boltovskoy, in press, fig. 15.18.

Solenosphaera chierchiae Brandt

(Pl. 2, Fig. 2)

Otosphaera polymorpha Haeckel; Nigrini and Moore, 1979, p. S9, pl. 1, fig. 5. Solenosphaera chierchiae Brandt; Boltovskoy, in press, fig. 15.22.

Family ACTINOMMIDAE Haeckel, 1862; emend. Riedel, 1967b 
Table 1. Radiolarian assemblages.

\begin{tabular}{|c|c|c|}
\hline Counting group & $\begin{array}{c}\text { Sample } \\
\text { 160-969A-1H-3, } \\
65-67 \mathrm{~cm}\end{array}$ & $\begin{array}{c}\text { Sample } \\
160-971 \mathrm{~B}-2 \mathrm{H}-\mathrm{CC}\end{array}$ \\
\hline (1) Collosphaerid group & 1.96 & 0.37 \\
\hline (5) Cladococcus group & 0.20 & $?$ \\
\hline (6-7) Hexacontium group & 1.76 & 10.97 \\
\hline (8) Thecospaera spp. & 0.20 & + \\
\hline (9) Other actinommids & 20.35 & 2.23 \\
\hline (11) Didymocyrtis spp. & $?$ & 0.19 \\
\hline (12) Dictyocoryne group & - & 1.30 \\
\hline (13) Hymeniastrum group & + & + \\
\hline (14) Porodiscus group A & 0.20 & 1.86 \\
\hline (15) Porodiscus group B & 0.20 & 1.12 \\
\hline (14-15) Porodiscus group & 0.78 & 3.53 \\
\hline (17) Spongaster spp. & + & 0.56 \\
\hline (18) Spongocore spp. & - & 0.19 \\
\hline (19) Spongodiscus group & - & 0.74 \\
\hline (20) Pyloniid group & 17.42 & 1.30 \\
\hline (21) Tholoniid group & - & 0.37 \\
\hline (23) Larcospira group & 0.39 & 0.37 \\
\hline (25) Pylospira group & 1.17 & 1.49 \\
\hline (26) Acanthodesmia group & 20.94 & 1.30 \\
\hline (27) Ceratospyris group A & 1.37 & 1.30 \\
\hline (28) Ceratospyris group B & - & 0.19 \\
\hline (29) Ceratospyris group C & 1.37 & 0.93 \\
\hline (30) Despospyris group & 0.20 & + \\
\hline (31) Nephrospyris spp. & 1.17 & 1.12 \\
\hline (32) Tholospyris group & 0.59 & + \\
\hline (35) Zygocircus group & 0.20 & 0.93 \\
\hline (37) Arachnocorys group & 0.78 & 2.23 \\
\hline (38) Arachnocorallium group & 2.15 & 21.75 \\
\hline (39) Ceratocyrtis group & 0.39 & 0.19 \\
\hline (40) Lophophaena group & 7.24 & 12.27 \\
\hline (42) Pseudodictyophimus group & - & 0.37 \\
\hline (43-44) Sethophormin group & + & 0.37 \\
\hline (45) Other plagoniids & - & 1.12 \\
\hline (46) Cornutella spp. & - & 1.49 \\
\hline (47) Eucecryphalus group & - & 3.16 \\
\hline (49) Eucyrtidium punctatum group & + & 2.23 \\
\hline$(48,50)$ Other eucyrtidium & 0.39 & 4.28 \\
\hline (53) Litharachnium group & - & 0.56 \\
\hline (55) Pterocanium group & 0.98 & 1.30 \\
\hline (57) Theocorys group & 0.39 & 0.93 \\
\hline (58) Other theoperids & - & 0.37 \\
\hline (59) Carpocanistrum group A & 5.68 & 1.49 \\
\hline (61) Anthocyrtidium group & 0.39 & 0.37 \\
\hline (62) Three-segmented pterocorythids & 0.78 & 1.67 \\
\hline (63) Botryostrobus group & 0.39 & 1.12 \\
\hline (64) Phormostichoartus group & $?$ & 0.56 \\
\hline (65) Siphocampe group & 0.19 & 0.19 \\
\hline (66) Spirocyrtis group & 0.19 & 1.30 \\
\hline (67) Cannobotryid group & - & 5.20 \\
\hline (68) Carpocanarium group & 0.59 & $?$ \\
\hline Not identified & 9.20 & 6.50 \\
\hline Total number of specimens counted & 511 & 538 \\
\hline
\end{tabular}

Note: The abundance of each taxon is expressed as a percentage. If a specimen was not identifiable because it was broken and incomplete, it was not counted. On the other hand, if a specimen was complete but its identification was hampered because of taxonomic uncertainties or inappropriate position in the slide, the specimen was counted but classified as "not identified." + = presence of very rare taxa that were not found during the counting; $-=$ these taxa were not observed; ? = identification is uncertain.

\section{Cladoccocus group}

Cladoccocus group; Riedel et al., 1985, p. 505, pl. 1, fig. 5.

\section{Hexacontium group}

\section{(Pl. 1, Fig. 1; Pl. 2, Figs. 3-4)}

Hexacontium group A; Riedel et al., 1985, p. 505, pl. 1, fig. 6a-c. Hexacontium group B; Riedel et al., 1985, p. 505, pl. 1, fig. 7a-c.

Remarks: Although both of the groups distinguished by Riedel et al. (1985) were encountered in our material, for most of the specimens it was difficult to count the number of pores across the half equator.

\section{Thecosphaera spp.}

(Pl. 2, Fig. 5)

Thecosphaera spp.; Riedel et al., 1985, p. 505, pl.1, fig. 8.

Thecosphaera inermis (Haeckel)

(Pl. 2, Fig. 5)

Thecosphaera inermis (Haeckel); Boltovskoy, in press, fig. 15.38.

\section{Other actinommids}

(P1. 1, Fig. 2; Pl. 2, Figs. 6-7)

Other actinommids; Riedel et al., 1985, p. 505, pl. 1, fig. 9a-d.

Remarks: The majority of morphotypes encountered in Sample 160-969A$1 \mathrm{H}-3,65-67 \mathrm{~cm}$ looks very similar to Hexacontium, but they do not display any medullary shell.

Family COCCODISCIDAE Haeckel, 1862; emend. Sanfilippo and Riedel, 1980

Didymocyrtis spp.

(Pl. 2, Fig. 8)

Ommatartus spp.; Dumitrica, 1973a, p. 834, pl. 6, fig. 6; pl. 11, fig. 7, 9 .

Peripanartium sp.; Sanfilippo et al., 1978, p. 756, pl. 1, fig. 6.

Didymocyrtis spp.; Riedel et al., 1985, p. 505, pl. 1, fig. 11a-e.

Family SPONGODISCIDAE Haeckel, 1862; emend. Riedel, 1967b

\section{Dictyocoryne group}

(Pl. 1, Figs. 3, 4; Pl. 2, Figs. 9-12)

Rhopalastrum spp.; Dumitrica, 1973a, p. 835, pl. 9, fig. 1, 3; pl. 11, fig. 4. Dictyocoryne group; Riedel et al., 1985, p. 505, pl. 2, fig. 1a-b.

Dictyocoryne profunda Ehrenberg

(Pl. 2, Fig. 9)

Dictyocoryne profunda Ehrenberg; Boltovskoy, in press, fig. 15.68.

Dictyocoryne truncatum (Ehrenberg)

(Pl. 1, Fig. 4; Pl. 2, Fig. 10)

Dictyocoryne truncatum (Ehrenberg); Boltovskoy, in press, fig. 15.69.

Hymeniastrum group

(Pl. 2, Figs. 13-15)

Hymeniastrum group; Riedel et al., 1985, p. 506, pl. 2, fig. 2a-b.

Hymeniastrum regulare (Haeckel)

(Pl. 2, Fig. 13)

Trigonastrum regulare Haeckel; Dumitrica, 1973a, p. 835, pl. 10, fig. 1-4; pl. 11 , fig. $1,3,5,8$

Trigonastrum regulare Haeckel; Sanfilippo et al., 1978, p. 757, pl. 1, fig. 11.

Remarks: It is worth noting that Dumitrica (1973a) found this species only in a very restricted interval of Site $128-3-6,80-100 \mathrm{~cm}$ (west of Crete). In order to elucidate the occurrence of this very distinctive species, it has been searched for in many late Neogene samples by Sanfilippo et al. (1978), but found only in Core 376-1 (west of Cyprus). This species is very rare and found only in sample 160-971B- $2 \mathrm{H}-\mathrm{CC}$.

\section{Hymeniastrum virchowii (Haeckel)}

$$
\text { (P1. 2, Fig. 14) }
$$

Amphirhopalum wirchowii (Haeckel); Dumitrica, 1973a, p. 835, pl. 9, fig. 2,4; pl. 11 , fig. 6 ; pl. 21, fig. 2-13. 
Porodiscus group

(Pl. 1, Fig. 5; Pl. 3, Figs. 1-4)

Porodiscus spp.; Dumitrica, 1973a, p. 835, pl. 8, fig. 1-6; pl. 9, fig. 6.

Remarks: Included are both groups A and B of Porodiscus described by Riedel et al. (1985). No morphotypes of group $C$ have been encountered in our material.

\section{Porodiscus group A}

(Pl. 3, Fig. 1)

Porodiscus group A; Riedel et al., 1985, p. 506, pl. 2, fig. 3a-b.

\section{Porodiscus group B \\ (P. 3, Figs. 2, 3)}

Porodiscus group B; Riedel et al., 1985, p. 506, pl. 2, fig. 4.

\section{Spongaster spp.}

(Pl. 1, Fig. 6; Pl. 3, Figs. 5-8)

Spongaster spp.; Riedel et al., 1985, p. 506, pl. 2, fig. 6a-c.

Spongaster sp (p); Sanfilippo et al., 1978, p. 756, pl. 1, fig. 7-9.

\section{Spongocore spp.}

(Pl. 3, Figs. 10-13)

Spongurus cylindricus Haeckel; Dumitrica, 1973a, p. 834, pl. 11, fig. 2; pl. 18, fig. 23.

Spongocore spp.; Riedel et al., 1985, p. 506, pl. 2, fig. 7

Spongurus cylindricus Haeckel; Van de Paverd, 1995, p. 149, pl. 40, fig. 1-9.

\section{Spongocore puella Haeckel}

(Pl. 3, Figs. 10, 11)

Spongocore puella Haeckel, 1887, p. 347, pl. 48, fig. 6.

Spongocore sp.; Riedel et al., 1985, pl. 2, fig. 7.

Spongocore puella Haeckel; Bjørklund and De Ruiter, 1987, fig. 3.15-16.

Spongodiscus group

(Pl. 3, Fig. 9)

Spongodiscus spp.; Dumitrica, 1973a, p. 834, pl. 9, fig. 5; pl. 10, fig. 5-6. Spongodiscus group; Riedel et al., 1985, p. 506, pl. 2, fig. 8 .

\section{Family PYLONIIDAE Haeckel, 1881 \\ Pyloniid group}

(Pl. 1, Fig. 7; Pl. 3, Fig. 14)

Tetrapyle (?) sp.; Dumitrica, 1973a, pl. 6, fig. 5.

Pyloniid group; Riedel et al., 1985, p. 506, pl. 2, fig. 9a-b.

\section{Family THOLONIIDAE Haeckel, 1887 \\ Tholoniid group \\ (Pl. 3, Fig. 15)}

Cubotholus sp.; Dumitrica, 1973a, pl. 7, fig. 3.

Tholoniid group; Riedel et al., 1985, p. 506, pl. 2, fig. 10.

\section{Amphitholus metallasson (Haeckel)}

(Pl. 3, Fig. 15)

Cubotholus sp.; Dumitrica, 1973a, pl. 7, fig. 3.

Amphitholus metallasson (Haeckel); Van de Paverd, 1995, p. 184, pl. 53, fig. 9-10, 12.

\section{Family LITHELIIDAE Haeckel, 1862 \\ Larcospira group}

Larcospira group; Riedel et al., 1985, p. 506, pl. 2, fig. 12.

Pylospira group

(Pl. 3, Figs. 16-18)
Pylospira group; Riedel et al., 1985, p. 509, pl. 2, fig. 14a-b.

Pylospira octopyle Haeckel

(Pl. 3, Fig. 16)

Pylospira octopyle Haeckel; Boltovskoy, in press, fig. 15.90.

Order NASSELLARIA

Family SPYRIDAE Ehrenberg, 1847

Acanthodesmia group

(Pl. 1, Fig. 8)

Acanthodesmia group; Riedel et al., 1985, p. 507, pl. 3, figs. 1a-b.

Acanthodesmia vinculata (Müller)

(P1. 1, Fig. 8)

Acanthodesmia vinculata (Müller); Dumitrica, 1973a, p. 841, pl. 28, figs. 1, 2.

\section{Ceratospyris group A}

(Pl. 4, Fig. 1)

Ceratospyris group A; Riedel et al., 1985, p. 507, pl. 3, fig. 2a-c.

\section{Ceratospyris group B}

(Pl. 4, Figs. 2, 3)

Ceratospyris (?) sp.; Dumitrica, 1973a, pl. 28, fig. 10.

Ceratospyris group B; Riedel et al., 1985, p. 507, pl. 3, figs. 3a-b.

Ceratospyris group $\mathbf{C}$

(Pl. 4, Figs. 4, 5)

Ceratospyris (?) spp.; Dumitrica, 1973a, p. 841, pl. 27, figs. 5, 6.

Ceratospyris group C; Riedel et al., 1985, p. 507, pl. 3, fig. 4.

\section{Desmospyris group}

(Pl. 4, Fig. 6)

Desmospyris group; Riedel et al., 1985, p. 507, pl. 3, figs. 5a-c.

\section{Nephrospyris spp.}

(Pl. 4, Fig. 7)

Nephrospyris spp.; Riedel et al., 1985, p. 507, pl. 3, fig. 6.

Nephrospyris renilla Haeckel

(Pl. 4, Fig. 7)

Nephrospyris renilla Haeckel; Dumitrica, 1973a, p. 841, pl. 28, fig. 8.

\section{Tholospyris group}

Tholospyris group; Riedel et al., 1985, p. 507, pl. 3, figs. 7a-b.

\section{Zygocircus group}

(Pl. 4, Figs. 8, 9)

Zygocircus group; Riedel et al., 1985, p. 507, pl. 3, figs. 10a-b.

$$
\text { Zygocircus productus productus (Hertwig) }
$$

(Pl. 4, Fig. 8)

Zygocircus productus (Hertwig); Dumitrica, 1973a, pl. 27, fig. 7 (only)

Zygocircus productus productus (Hertwig); Goll, 1979, p. 382, pl. 2, fig. 3.

Zygocircus productus forma productus (Hertwig); Van de Paverd, 1995, p. 201, pl. 62, fig. 14.

\section{Zygocircus productus piscicaudatus Popofsky}

(Pl. 4, Fig. 9)

Zygocircus productus (Hertwig); Dumitrica, 1973a, pl. 27, fig. 8 (only). 
Zygocircus productus piscicaudatus Popofsky; Goll, 1979, p. 382, pl. 2, figs. $1,2$.

Zygocircus productus forma piscicaudatus Popofsky; Van de Paverd, 1995, p. 202 , pl. 62 , figs. 11,13 .

Family PLAGONIIDAE Haeckel, 1881; emend. Riedel, 1967b

\section{Arachnocorys group}

(Pl. 4, Fig. 10)

Arachnocorys group; Riedel et al., 1985, p. 508, pl. 3, figs. 12a, b.

\section{Arachnocorys circumtexta Haeckel}

$$
\text { (Pl. 4, Fig. 10) }
$$

Arachnocorys circumtexta Haeckel, 1862, p. 304, pl. 6, figs. 9-11.

\section{Arachnocorallium group \\ (Pl. 4, Figs. 11-15)}

Arachnocorallium group; Riedel et al., 1985, p. 508, pl. 3, figs. 13a-c.

\section{Ceratocyrtis group}

(Pl. 4, Fig. 17)

Ceratocyrtis group; Riedel et al., 1985, p. 508, pl. 3, figs. 14a-c.

\section{Lophophaena group \\ (Pl. 4, Figs. 18-21)}

Lophophaena group; Riedel et al., 1985, p. 508, pl. 3, figs. 15a-c.

\section{Pseudodictyophimus group}

(Pl. 4, Fig. 22)

Pseudodictyophimus group; Riedel et al., 1985, p.508, pl. 3, figs. 17a, b.

\section{Sethophormin group

$$
\text { (P1. 4, Fig. 23) }
$$

Sethophormin group A; Riedel et al., 1985, p. 508, pl. 4, figs. 1a, b. Sethophormin group B; Riedel et al., 1985, p. 508, pl. 4, figs. 2a, b.

\section{Other plagoniids}

(Pl. 4, Fig. 24)

Other plagoniids; Riedel et al., 1985, p. 508, pl. 4, figs. 3a-c.

\section{Clathrocorys teuscheri Haeckel}

(Pl. 4, Fig. 24)

Clathrocorys teuscheri Haeckel; Bjørklund and De Ruiter, 1987, fig. 6.21. Clathrocorys teuscheri Haeckel; Boltovskoy, in press, fig. 15.112.

Family THEOPERIDAE Haeckel, 1881; emend. Riedel, 1967b Cornutella spp.

$$
\text { (Pl. 5, Figs. 1-3) }
$$

Cornutella spp.; Riedel et al., 1985, p. 509, pl. 4, fig. 4.

$$
\text { Cornutella profunda Ehrenberg }
$$$$
\text { (Pl. 5, Figs. 2, 3) }
$$

Cornutella profunda Ehrenberg; Boltovskoy, in press, fig. 15.127.

\section{Eucecryphalus group}

(Pl. 1, Figs. 9, 10; Pl. 5, Figs. 4, 5)

Eucecryphalus group; Riedel et al., 1985, p. 509, pl. 4, figs. 5a-b.

\section{Eucyrtidium punctatum (Ehrenberg) group (Pl. 5, Figs. 6-8)}

Stichopterygium anomalum (Haeckel); Dumitrica, 1973a, p. 838, pl. 27, fig. 11.
Eucyrtidium anomalum (Haeckel); Sanfilippo et al., 1978, p. 756, pl. 1, figs. $1,2$.

Eucyrtidium punctatum (Ehrenberg) group; Sanfilippo et al., 1978, p. 756, pl. 1 , fig. 3 .

Eucyrtidium punctatum (Ehrenberg) group; Riedel et al., 1985, p. 509, pl. 4, fig. 7.

Other Eucyrtidium

(Pl. 1, Fig. 11; Pl. 5, Figs. 9-11)

Eucyrtidium spp.; Dumitrica, 1973a, p. 839, pl. 27, figs. 1, 2.

Other Eucyrtidium; Riedel et al.,1985, p. 509, pl. 4, figs. 8a, b.

Eucyrtidium cienkowskii Haeckel group; Riedel et al., 1985, p. 509, pl. 4, fig. 6.

Eucyrtidium acuminatum (Ehrenberg)

(Pl. 1, Fig. 11; Pl. 5, Fig. 9)

Eucyrtidium acuminatum (Ehrenberg); Nigrini and Moore, 1979, p. N61, pl. 24 , figs. $3 \mathrm{a}-\mathrm{b}$.

Eucyrtidium acuminatum (Ehrenberg); Bjørklund and De Ruiter, 1987, fig. 4.20-23.

Eucyrtidium acuminatum (Ehrenberg); Boltovskoy, in press, fig. 15.137

\section{Litharachnium group}

(Pl. 5, Fig. 12)

Litharachnium group; Riedel et al., 1985, p. 509, pl. 4, fig. 11.

\section{Pterocanium group \\ (Pl. 1, Fig. 12)}

Pterocanium group; Riedel et al., 1985, p. 509, pl. 4, fig. 13.

\section{Theocorys group}

Theocorys group; Riedel et al., 1985, p. 510, pl. 4, fig. 15.

Other theoperids

(Pl. 1, Fig. 13; Pl. 5, Figs. 13, 14)

Other theoperids; Riedel et al., 1985, p. 510, pl. 5, fig. 1a-3.

Lithostrobus hexagonalis Haeckel (Pl. 5, Fig. 13)

Lithostrobus hexagonalis Haeckel; Boltovskoy, in press, fig. 15.141.

\section{Lipmanella bombus (Haeckel)}

(Pl. 1, Fig. 13)

Lipmanella bombus (Haeckel); Boltovskoy, in press, fig. 15.133.

Lipmanella dictyoceras Haeckel

(Pl. 5, Fig. 14)

Lipmanella dictyoceras Haeckel; Boltovskoy, in press, fig. 15.134.

Family CARPOCANIIDAE Haeckel, 1881; emend. Riedel, 1967b

Carpocanistrum group A

(Pl. 5. Figs. 17, 18)

Carpocanistrum group A; Riedel et al., 1985, p. 510, pl. 5, fig. 2.

Family PTEROCORYTHIDAE Haeckel, 1881; emend. Riedel, 1967b Anthocyrtidium group

(Pl. 5, Fig. 15)

Anthocyrtidium group; Riedel et al., 1985, p. 510, pl. 5, fig. 5.

Three-segmented pterocorythids

(Pl. 1, Figs. 14, 15; Pl. 5, Fig. 16)

Lamprocyclas group; Riedel et al., 1985, p. 510, pl. 5, figs. 6a, b. 
Family ARTOSTROBIIDAE Riedel, 1967a

Botryostrobus group

(Pl. 5, Figs. 19, 20)

Botryostrobus group; Riedel et al., 1985, p. 510, pl. 5, fig. 7.

\section{Phormostichoartus group}

(Pl. 5, Figs. 21, 22)

Phormostichoartus group; Riedel et al., 1985, p. 510, pl. 5, figs. 10a, b.

\section{Siphocampe group}

(Pl. 5, Figs. 23, 24)

Siphocampe group; Riedel et al., 1985, p. 510, pl. 5, figs. 8a, b.

\section{Spirocyrtis group}

(Pl. 5, Figs. 25, 26)

Spirocyrtis group; Riedel et al., 1985, p. 511, pl. 5, fig. 9.

Family CANNOBOTRYIDAE Haeckel, 1881; emend. Riedel, 1967b Cannobotryid group

(Pl. 5, Figs. 27-30)

Cannobotryid group; Riedel et al., 1985, p. 511, pl. 5, figs. 11a, b.

\section{INCERTAE CEDIS \\ Carpocanarium group}

Carpocanarium group; Riedel et al., 1985, p. 511, pl. 5, figs. 4a, b.

\section{SILICOFLAGELLATE RESULTS AND SYSTEMATICS}

The results of extensive stratigraphic research on silicoflagellates from the islands of Crete, Milos, and Aegina (Aegean Sea, Greece) have been recently published by Frydas (1996). Seven new Neogene acme-subzones and a new Pleistocene acme-subzone (Octactis pulchra) were defined therein. The exact position of the upper boundary of the latter acme-subzone has not yet been defined in these areas because of the absence of suitable outcrops of middle late Pleistocene age.

Three hundred silicoflagellate specimens were counted from the sample 160-971B-2H-CC. The relative abundance (\%) of existing species is as follows, where A: Abundant (16-20\%), C: Common (11-15\%), F: Frequent (4-10\%), R: Rare (1-3\%):

\footnotetext{
Dictyocha aculeata aculeata (A),

D. calida ampliata $(\mathrm{C})$,

D. delicata $(\mathrm{C})$,

D. fibula $(\mathrm{F})$,

D. messanensis $(\mathrm{F})$,

D. perlaevis perlaevis $(\mathrm{F})$,

D. stapedia aspinosa $(\mathrm{C})$,

D. stapedia stapedia $(\mathrm{C})$,

Distephanus octogonus $(\mathrm{R})$
}

The assemblage is assigned to the Dictyocha aculeata aculeata Zone, which is defined as the interval following the last occurrence of Bachmannocena quadrangula (middle late Pleistocene, Bukry, 1981, 1995). The type horizon of this zone is correlated with the following middle late Pleistocene biozones of calcareous nannoplankton: NN20/21 of Martini (1971), CN14b/15 of Okada and Bukry (1980) and MNN20/21 of Rio et al. (1990). The Dictyocha aculeata aculeata Zone can be correlated to:

1. The D. aculeta Zone of Dumitrica (1973b, 1973c), from the Mediterranean Sea and the southwestern Pacific Ocean;
2. The homonymous Zone of Bukry $(1979,1980)$, from the Site 397 off northwest Africa and from the Leg 54, in the eastern Equatorial Pacific;

3. The D. messanensis aculeata Zone of Martini (1990), Leg 112, from the eastern Pacific off Peru;

4. The D. messanensis aculeata Zone of Locker (1995), from off southern Chile.

Silicoflagellate Systematics

Genus DICTYOCHA Ehrenberg, 1837

Dictyocha aculeata aculeata (Lemmermann, 1901)

(Pl. 6, Figs. 7-16)

1901: Dictyocha aculeata aculeata Lemmermann, p. 261, pl. 11, figs. 1, 2.

1935: Dictyocha fibula var. aculeata Lemmermann; Frenguelli, pl. 13, figs. $1-9$.

1968: Dictyocha epiodon Ehrenberg; fide Loeblich et al., p. 90, fig. 15.

1970: Dictyocha aculeata var. aculeata Lemmermann; Ling, p. 91, pl. 18 , figs. 11-13.

1973b: Dictyocha aculeata (Lemmermann); Dumitrica, p. 907, pl. 9, figs. 510.

1973c: Dictyocha aculeata (Lemmermann); Dumitrica, p. 849, pl. 4, figs. 911.

1973: Dictyocha epiodon Ehrenberg; Bukry and Foster, p. 826, pl. 2, figs. 7-

1974: Dictyocha epiodon Ehrenberg; Locker, p. 634, pl. 1, figs. 1, 4, 5, 7, 8. 1975: Dictyocha aculeata (Lemmermann); Perch-Nielsen, p. 686, pl. 5, figs. $3,4$.

1976: Dictyocha epiodon Ehrenberg; Poelchau, p. 170, pl. 1, figs. e, f; pl. 4, figs. 7,8 .

1977: Dictyocha aculeata (Lemmermann); Bukry, p. 921.

1980: Dictyocha aculeata aculeata (Lemmermann); Bukry, pp. 549-552, pl. 1 , figs. $1-3$.

1986: Dictyocha messanensis ssp. aculeata (Lemmermann) f. aculeata; Locker and Martini, p. 904, pl. 3, figs. 11, 12.

1990: Dictyocha messanensis ssp. aculeata (Lemmermann) f. aculeata; Martini, p. 169, fig. 2.

1995: Dictyocha messanensis ssp. aculeata (Lemmermann); Locker, p. 233, fig. 2.

1995: Dictyocha aculeata aculeata (Lemmermann); Bukry, p. 4, fig. 4(B)

Remarks: This important Quaternary warm-water species is characterized by the additional basal horns between the lateral bars and the sustaining spines, as well as by the presence of further additional spines on the basal ring and apical apparatus (Dumitrica, 1973b; Bukry, 1995). Two varieties of D. aculeata aculeata can be distinguished in our material, the large variety being almost one and a half times as large as the small one. In addition to these two varieties one pentagonal form was also observed (Pl. 1, fig. 15). According to Dumitrica (1973b) this taxon seems to occur exclusively in the Quaternary.

Dictyocha calida ampliata Bukry, 1979 (Pl. 6, Figs. 1-6)

1979: Dictyocha calida ampliata n. subsp.; Bukry, p. 982-983, pl. 2, figs. 1, $2,9$.

Remarks: According to Bukry (1979) D. calida ampliata is distinguished from $D$. calida calida by more extreme elongation of the portals. The ratio of the external width of the ring (X1) to the width of apical structure (X2) as measured perpendicularly to the bar allows a proportional comparison of elongation of portals between $D$. calida calida Poelchau (1976) and $D$. calida ampliata (Bukry, 1979). The ratio X1/X2 of D. calida ampliata in our material varies from 2.50 to 2.71 , based on measurements made on 20 specimens, whereas the same ratio for Poelchau's $D$. calida calida varies from 2.07 to 2.39. D. calida ampliata is known to date from upper Pliocene to Holocene sediments only (Perch-Nielsen, 1985).

Dictyocha delicata (Bukry, 1982)

(Pl. 7, Figs. 4-6)

1976: Dictyocha perlaevis delicata; Bukry, p. 724, pl. 1, figs. 5-10. 1982: Dictyocha delicata (Bukry); Bukry, p. 432, pl. 2, fig. 7. 
1994: Dictyocha delicata (Bukry); Frydas, p. 486, pl. 1, fig. 8.

Remarks: Dictyocha delicata is found in smaller numbers near Heraklion (Crete) in the locality of Gournes (Frydas, 1994). The silicoflagellate assemblages from the section Gournes belong to the Dictyocha neonautica acmeSubzone of Piacenzian age (Frydas, 1996). The stratigraphic distribution of $D$. delicata extends from the upper Pliocene through the Quaternary. It is most common in the lower Quaternary levels of Sites 157 and 321 (southeastern Pacific), but it is rare in the upper Quaternary levels of Site 321 (Bukry, 1976).

\section{Dictyocha fibula Ehrenberg, 1839}

(Pl. 7, Figs. 18, 19)

1839: Dictyocha fibula Ehrenberg; fide Loeblich et al., 1968, p. 90, pl. 9, figs. 7-12.

1973b: Dictyocha fibula Ehrenberg; Dumitrica, p. 906, pl. 3, figs. 7, 9; pl. 4, fig. 8 ; pl. 5, figs. $1-5,8$; pl. 6 , figs. $1,2,4,5,7,8$; pl. 9 , fig. 1 .

1973: Dictyocha fibula Ehrenberg; Bukry and Foster, p. 826-827, pl. 2, fig. 9; pl. 3, figs. 1, 12 .

1990: Dictyocha fibula Ehrenberg; Frydas, p. 96, pl. 1, fig. 13.

Remarks: According to Dumitrica (1973b), the Quaternary forms possess a squarish or slightly rhombic basal ring, with rounded corners, rather long blunt radial horns, and short sustaining spines. Locker's (1974) taxonomy for D. fibula is not accepted herein.

Dictyocha messanensis Haeckel in Peters, 1860 (Pl. 6, Figs 17, 18)

1860: Dictyocha messanensis Haeckel in Peters; fide Loeblich et al., 1968, p. 103-104, pl. 17, figs. 24-27.

1972: Dictyocha fibula Ehrenberg var. messanensis (Haeckel); Ling, p. 161, pl. 25 , figs. 18,19 .

1973b: Dictyocha messanensis Haeckel; Dumitrica, p. 907, pl. 8, figs. 8-13; pl. 9 , figs. $2-4$.

1973c: Dictyocha messanensis Haeckel; Dumitrica, p. 849, pl. 4, figs. 7, ?5, 6, 7, 8; ?pl.3, figs. 13-15.

1975: Dictyocha messanensis Haeckel; Perch-Nielsen, p. 686, pl. 5, figs. 13, 14.

1976: Dictyocha messanensis Haeckel; Martini, p. 443, pl. 1, fig. 9; pl. 2, figs. 2a, b.

1986: Dictyocha messanensis aspinosa (Bukry); Locker and Martini, p. 904, pl. 3 , figs. $3-5$; pl. 12 , fig. 2 .

1986: Dictyocha messanensis messanensis fa. aspinosa (Bukry);

Locker and Martini, p. 904, pl. 3, figs. 1, 9.

1995: Dictyocha messanensis Haeckel; McCartney et al., p. 147, pl. 3, figs. $12,13$.

Remarks: Dictyocha messanensis is a polytypic and polymorphic species. It can be distinguished from $D$. aculeata not only by the lack of additional basal horns, but also by its smaller size. D. messanensis is the most frequent species in the Quaternary of Sites 127 and 128 (Eastern Mediterranean) and is regarded as a warm-water species (Dumitrica, 1973b). Small, rhomboidal, more delicate forms were found in the material studied, which according to Dumitrica (1973c), probably indicate a great amount of sapropel. Species without apical or sustaining spines are known from Pleistocene and Holocene sediments (Perch-Nielsen, 1975).

Dictyocha perlaevis perlaevis Frenguelli, 1951

(Pl. 7, Figs. 20, 21)

1951: Dictyocha perlaevis Frenguelli, p. 279, figs. 4b, c.

1973: Dictyocha fibula Ehrenberg; Bukry and Foster, p. 826, pl. 3, fig. 1.

1975: Dictyocha fibula perlaevis (Frenguelli); Bukry, p. 855, pl. 3, fig. 5.

1978: Dictyocha perlaevis Frenguelli; Stradner and Bachmann, p. 806, pl. 1, figs. 5, 6, 9-11.

1979: Dictyocha perlaevis perlaevis Frenguelli; Bukry, p. 984, pl. 3, figs. 6-11. 1986: Dictyocha messanensis ssp. stapedia (Haeckel) f. stapedia; Locker and Martini, p. 905, pl. 3, figs. 6, 7 .

1990: Dictyocha perlaevis perlaevis Frenguelli; Frydas, p. 97, pl. 1, fig. 14.

Remarks: D. perlaevis perlaevis is a warm-water species most commonly found in Pliocene and Quaternary assemblages. D. perlaevis perlaevis differs from $D$. fibula by the long size of the basal ring and the pentals of the minor axis. D. perlaevis perlaevis is present in lesser numbers in our material.

Dictyocha stapedia aspinosa Bukry, 1976

(Pl. 7, Figs. 1-3, 7-10)

1976: Dictyocha stapedia aspinosa; Bukry, p. 724 (in part), pl. 2, figs. 7, 8. 1990: Dictyocha stapedia aspinosa; Frydas, p. 97, pl. 1, figs. 15, 16.

Remarks: The most significant criterion by which to distinguish $D$. stapedia aspinosa from Dictyocha messanensis is the distinctive basal spikes, which were also used by Haeckel (1887) to distinguish the two species. D. stapedia is widespread in all warm seas (Bukry, 1976). Its stratigraphic range extends from upper Pliocene to the Holocene.

\section{Dictyocha stapedia stapedia Haeckel, 1887}

(P1. 7, Figs. 14-17)

1887: Dictyocha stapedia; Haeckel, p. 1561, pl. 101, figs. 10-12.

1887: Dictyocha rhombus; Haeckel, p. 1562.

1901: Dictyocha rhombus Haeckel; Lemmermann, pl. 11, fig. 3

1976: Dictyocha stapedia stapedia Haeckel; Bukry, p. 724, pl. 3, figs. 1-7.

1986: Dictyocha messanensis Haeckel; Frydas, p. 8, pl. 5, fig. 4.

Remarks: D. stapedia stapedia's longer radial spines along the major axis distinguish it from $D$. stapedia aspinosa. D. messanensis differs from $D$. stapedia stapedia in having a short apical bar. D. stapedia stapedia is a species with a wide geographic distribution whose range extends from the upper Pliocene to the Holocene.

Genus DISTEPHANUS Stohr, 1880

Distephanus octogonus (Ehrenberg, 1843)

(Pl. 7, Figs. 11-13)

1843: Mesocena? octogona Ehrenberg; fide Loeblich et al., 1968, p. 129, pl. 27, fig. 6).

1846: Mesocena binonaria Ehrenberg; fide Loeblich et al., 1968, p. 127, pl. 27, fig.1.

1846: Mesocena bioctonaria Ehrenberg; fide Loeblich et al., 1968, p. 127, pl. 27, fig. 2.

1973b: Distephanus octogonus (Ehrenberg); Dumitrica, p. 908, pl. 12, fig. 16-19.

1974: Octactis? bioctonaria (Ehrenberg); Locker, p. 636, pl. 2, fig. 5-7.

1978: Distephanus octogonus (Ehrenberg); Stradner and Bachmann, p. 807, pl. 2, fig. 16.

1990: Distephanus bioctonarius f. binonarius (Ehrenberg) nov. comb; Martini, p. $166-167$, pl. 3 , figs. 5,6 .

1990: Distephanus bioctonarius f. bioctonarius (Ehrenberg) nov. comb; Martini, p. 167, pl. 3, figs. 3,4 .

Remarks: Eight-rayed forms of D. octogonus (Pl. 7, Fig. 13) are the common morphotypes in our material. Only two specimens with nine spines were found (Pl. 7, Figs. 11, 12). According to Dumitrica (1973b), D. octogonus appears to be restricted to the Quaternary (DSDP Sites 127 and 128 of the Eastern Mediterranean). This species occurs rarely in the Quaternary sediments of the Eastern Pacific (Bukry and Foster, 1973).

\section{RADIOLARIAN PALAEOECOLOGICAL INTERPRETATION}

The radiolarian assemblage in Sapropel S5 records an abundant, well-preserved and diverse fauna, which reflects accumulation below eutrophic waters (Casey, 1993). More particularly, it is dominated by Arachnocorallium gr. (22\%), a counting-group that was correlated by Riedel et al. (1985) to Peridinium spinipes Haeckel, as studied by Casey et al. (1979a, 1979b). In peak abundances of polycystine radiolarians, which occur at depths between 100 and $200 \mathrm{~m}$ in the oligotrophic, open ocean Sargasso Sea, this species was found to be dominant. The same species was also found to be dominant in much more eutrophic waters, such as the southern Californian and the Gulf 
Stream where the densities of living radiolarians peak in the upper $100 \mathrm{~m}$ of the water column (Casey et al., 1979a, 1979b). According to Casey et al. (1979a) the abundance of Peridinium spinipes in sediments can be regarded as an indicator of high productivity in the overlying waters. High primary production during accumulation of Sapropel S5 is independently supported by geochemical studies. Among the nine youngest sapropels of the Eastern Mediterranean, the lightest values of nitrogen isotope ratios were measured in this particular sapropel (Calvert et al., 1992). High productivity resulted in significantly high flux of organic matter in the S5 sapropel; this is recorded in the highest levels of organic carbon content preserved in it (Ten Haven et al., 1987; Fontugne and Calvert, 1992).

Ecological studies of living radiolarians suggest that many are restricted to certain depths or ecological niches. These are mainly feeding niches, reflecting different feeding strategies, but it is clear that some radiolarians may be involved in more than one feeding activity (Casey et al., 1979a; Anderson, 1983, 1993). Most radiolarian species prefer shallow-water niches and diversity decreases with depth (McMillen and Casey, 1978), but radiolarians have been reported living in waters as deep as $5000 \mathrm{~m}$ (Takahashi and Honjo, 1981). Shallow-water niches are mainly occupied by polycystines with symbiotic zooxanthellae (algae). Many of the near-surface-dwelling radiolarians contain algal symbionts in their cytoplasm, which to a large extent could provide the radiolarians' food requirements (Anderson, 1983, 1993). Among the taxa identified in our material, the collosphaerid group, Hexacontium gr., other actinommids, pyloniid gr., Acanthodesmia gr., Dictyocoryne gr., and Spongaster spp. would fall in this group (the latter two are more abundant in subsurface waters, see below). They represent a significant part of the radiolarian assemblage in the sapropel S5 (18\%), but they form almost two thirds of the assemblage $(62.5 \%)$ in Sample 160-969A-1H-3, 65-67 cm.

Depth profiles of radiolarian densities in the North Pacific and equatorial Atlantic (Kling, 1979; Dworetzky and Morley, 1987) establish that although many species are restricted to discrete depth zones (which is an indication of preferred habitats for those species) peak abundances of polycystines as a whole would in general coincide with a chlorophyll-maximum zone occurring at subsurface depths $(50-100 \mathrm{~m})$. This niche would be mainly occupied by herbivorous polycystines involved in the grazing food chain and fed essentially upon the micro- and nannophytoplankton. According to Casey et al. (1979a), Arachnocorallium gr. would belong to this niche. The presence of a deep chlorophyll-maximum layer is a characteristic feature of most oceanic waters, which may be stratified, and has also been detected in the Eastern Mediterranean (Abdel-Moati, 1990). More particularly, vertical profiles of chlorophyll- $\alpha$ and biogenic silica (diatoms) measured off the Egyptian coast displayed positive peaks during early summer, at depths of approximately $100 \mathrm{~m}$, at the base of the euphotic zone.

Despite the limited amount of data relevant to the vertical distribution of radiolarians in the water column, some ecological information can be drawn for a certain number of them. Radiolarians such as Eucecryphalus gr., Litharachnium gr., Phormostichoartus gr., Dictyocoryne gr., Spongodiscus gr., Ceratospyris gr. A, Spongaster spp., Didymocyrtis spp., Larcospira gr., Anthocyrtidium gr., and Spongocore spp. are known to be present in peak abundances at or near (above or below) the level of chlorophyll- $\alpha$ maxima (Kling, 1979; Dworetzky and Morley, 1987). They represent more than $9 \%$ of the radiolarian assemblage in Sapropel S5 and only $2.15 \%$ in Sample 160-969A-1H-3, 65-67 cm.

Deep-water habitats were also occupied during the accumulation of sapropel S5, as indicated by the significant abundance of deepdwelling radiolarians (Cornutella spp., 1.5\%). These habitats belong to the detritivore niche of polycystines - which feed upon suspended and settling dead or detrital material-and would be represented by Eucecryphalus gr., Cornutella spp., and Litharachnium gr. in our ma- terial (Casey et al., 1979a). Radiolarians of this niche are absent from above the sapropel S5, but they are well-represented in the sapropel itself $(5.2 \%)$.

All the above would suggest that during accumulation of Sapropel S5 radiolarians occupied ecological niches at various depths of the water column. The composition of the radiolarian assemblages indicates much stronger and better developed subsurface chlorophyllmaxima during accumulation of the sapropel S5. These results are in agreement with the paleoecological information yielded by planktonic foraminifers and calcareous nannofossils from this sapropel, which point to increased primary production confined to a chlorophyll maximum during its accumulation (Rohling and Gieskes, 1989; Castradori, 1993). However, after the end of the period of eutrophication (sapropel S5) the spectrum of radiolarian habitats was substantially reduced. This is reflected in the relative abundance of near-surface dwelling polycystines, whose energy needs are substantially supported by their symbionts (Anderson, 1993).

\section{DISCUSSION AND CONCLUSIONS}

Finding abundant and well-preserved siliceous microfossil assemblages in the Mediterranean sediments has a double importance: (1) in terms of biological productivity and (2) in terms of silica preservation on the seafloor and in the sediments.

Dissolution of diatom and radiolarian tests is a common phenomenon in seawater because of its undersaturation in silica (Berger, 1968; Schrader, 1971). This phenomenon appears to be much more intense in the Mediterranean Sea because of its very low silica content (Krom et al., 1991a). SEM observations of radiolarian tests from the surface of the Mediterranean seafloor display clear indications of dissolution (Caulet, 1972). Normally, in the Mediterranean, silica dissolution is very active in the upper $30 \mathrm{~cm}$ of the surface sediments; radiolarian tests have not been observed under this depth, apart from their exceptional preservation in some sapropel layers (Caulet, 1974). However, recent studies in small anoxic basins of the Eastern Mediterranean did establish that radiolarian preservation is very good in sediments that accumulated in the anoxic and hypersaline waters of these small protected basins (Bjørklund and De Ruiter, 1987). Similarities could be drawn to explain the exceptional preservation of opal assemblages in the S5 sapropel of Site 971 (Holes B and C; Shipboard Scientific Party, 1996b, Fig. 8), which accumulated in the moat of the Napoli mud volcano. However, the protection provided by this depression can only account for silica preservation to a certain extent. Siliceous microfossils have not been reported from the sediments in the same holes overlying the sapropel S5, not even in the well-expressed S1 layer. Hence, biogenic silica preserved in S5 has recorded a real event of particular significance.

It is possible that this particular event was the result of excessive discharge of the Nile river, which provided abundant nutrients for the Eastern Mediterranean. In present times, phosphorus appears to be the main biolimiting nutrient in the Eastern Mediterranean (Krom et al., 1991b). Also, because of the low values of silica in the Mediterranean, the limited availability of this element important for the healthy growth of diatoms (Ludwig and Volcani, 1986) and silicoflagellates (Lipps, 1970) would certainly limit the bloom of siliceous phytoplankton. Both phosphorus and silica could have been supplied in excess by the Nile river, after the monsoonal precipitations in eastern Africa.

Excessive bloom of diatom mats and their high rate of accumulation (Sancetta, 1994) would not only preserve the organic matter, but would also preserve biogenic silica from dissolution. Indeed, within areas of high productivity, especially of excessive diatom production with their subsequent export flux onto the seafloor, dissolution of sil- 
ica is substantially reduced, as deep waters become successively enriched in this element (Caulet, 1977; Qiu et al., 1993).

Radiolarian shell construction appears not to be limited by low levels of silica availability in the seawater. Moreover, symbiont-bearing radiolarians may be more competitive in absorbing silica even if it is available in very low quantities, since part of the substantial source of energy required may be supplied by the symbiont primary productivity (Anderson, 1996). The absence of preserved siliceous phytoplankton and the relative abundance of symbiont-bearing radiolarians above the sapropel S5, therefore, could be interpreted as the result of a drastic reduction in silicic acid and other biolimiting nutrients provided by the Nile into the Mediterranean.

\section{ACKNOWLEDGMENTS}

T. Danelian acknowledges financial support from the Royal Society of Edinburgh (BP Grant) and K. Bjørklund for his assistance during the early stages of this study. The authors are grateful to P. Dumitrica and K. Bjørklund for critical review of the manuscript. Many thanks to Y. Cooper for assistance with photography.

\section{REFERENCES}

Abdel-Moati, A.R., 1990. Particulate organic matter in the subsurface chlorophyll maximum layer of the Southeastern Mediterranean. Oceanol. Acta, 13:307-315.

Anastasakis, G.K., and Filippas, D., 1990. The most recent phase of enhanced productivity in the Aegean-East Mediterranean Sea. Proc. III Panhellenic Congr. Oceanogr. Fish., Athens, 147-154.

Anderson, O.R., 1983. Radiolaria: New York (Springer-Verlag).

1993. The trophic role of planktonic foraminifera and radiolaria. Mar. Microb. Food Webs, 7:31-51.

, 1996. The physiological ecology of planktonic Sarcodines with applications to paleoecology: patterns in space and time. J. Euk. Microbiol., 43:261-274.

Berger, W.H., 1968. Radiolarian skeletons: solution at depths. Science, 159:1237-1239.

Bjørklund, K.R., and De Ruiter, R., 1987. Radiolarian preservation in eastern Mediterranean anoxic sediments. Mar. Geol., 75:271-281.

Boltovskoy, D., in press. Radiolaria Polycystina. In Boltovskoy, D. (Ed.), South Atlantic Zooplankton: Amsterdam (SPB Academic Publ.).

Brasier, M.D., 1995. Fossil indicators of nutrient levels. 1: Eutrophication and climate change. In Bosence, D.W., and Allison, P.A. (Eds.), Marine Palaeoenvironmental Analysis from Fossils. Geol. Soc. Spec. Publ. London, 83:113-132.

Bukry, D., 1975. Silicoflagellate and coccolith stratigraphy, Deep Sea Drilling Project, Leg 29. In Kennett, J.P., Houtz, R.E., et al., Init. Repts. DSDP, 29: Washington (U.S. Govt. Printing Office), 845-872.

1976. Silicoflagellate and coccolith stratigraphy, southeastern Pacific Ocean, Deep Sea Drilling Project Leg 34. In Yeats, R.S., Hart, S.R., et al., Init. Repts. DSDP, 34: Washington (U.S. Govt. Printing Office), $715-735$.

— 1977 . Coccolith and silicoflagellate stratigraphy, central north Atlantic Ocean, Deep Sea Drilling Project Leg 37. In Aumento, F., Melson, W.G., et al., Init. Repts. DSDP, 37: Washington (U.S. Govt. Printing Office), 917-927.

_ 1979. Comments on opal phytoliths and stratigraphy of Neogene silicoflagellates and coccoliths at Deep Sea Drilling Project Site 397 off northwest Africa. In Luyendyk, B.P., Cann, J.R., et al., Init. Repts. DSDP, 49: Washington (U.S. Govt. Printing Office), 977-1009.

, 1980. Silicoflagellate biostratigraphy and paleoecology in the eastern equatorial Pacific, Deep Sea Drilling Project Leg 54. In Rosendahl, B.R., Hekinian, R., et al., Init. Repts. DSDP, 54: Washington (U.S. Govt. Printing Office), 545-573.

, 1981. Synthesis of silicoflagellate stratigraphy for Maestrichtian to Quaternary marine sediments. In Warme, T.E., Douglas, R.C., and Winterer, E.L. (Eds.), The Deep Sea Drilling Project: A Decade of Progress. Spec. Publ.-Soc. Econ. Paleontol. Mineral., 32:433-444.
, 1982. Cenozoic silicoflagellates from offshore Guatemala, Deep Sea Drilling Project Site 495. In Aubouin, J., von Huene, R., et al., Init. Repts. DSDP, 67: Washington (U.S. Govt. Printing Office), 425-445.

, 1995. Silicoflagellates and their geological application. Open-File Rep.-U.S. Geol. Surv., 95-260:1-27.

Bukry, D., and Foster, J.H., 1973. Silicoflagellate and diatom stratigraphy, Leg 16, Deep Sea Drilling Project. In van Andel, T.H., Heath, G.R., et al., Init. Repts. DSDP, 16: Washington (U.S. Govt. Printing Office), 815871.

Calvert, S.E., Nielsen, B., and Fontugne, M.R., 1992. Evidence from nitrogen isotope ratios for enhanced productivity during the formation of eastern Mediterranean sapropels. Nature, 359:223-225.

Casey, R.E., 1993. Radiolaria. In Lipps, J.H. (Ed.), Fossil Prokaryotes and Protists: Boston (Blackwell Scientific), 249-284.

Casey, R.E., Gust, L., Leavesley, A., Williams, D., Reynolds, R., Duis, T., and Spaw, J.M., 1979a. Ecological niches of radiolarians, planktonic foraminiferans and pteropods inferred from studies on living forms in the Gulf of Mexico and adjacent waters. Trans. Gulf Coast Assoc. Geol. Soc., 29:216-223.

Casey, R.E., Spaw, J.M., Kunze, F., Reynolds, R., Duis, T., McMillen, K., Pratt, D., and Anderson, V., 1979b. Radiolarian ecology and the development of the radiolarian component in Holocene sediments, Gulf of Mexico and adjacent seas with potential paleontological applications. Trans. Gulf Coast Assoc. Geol. Soc., 29:228-237.

Castradori, D., 1993. Calcareous nannofossils and the origin of eastern Mediterranean sapropels. Paleoceanography, 8:459-471.

Caulet, J.P., 1972. Premières observations sur la dissolution progressive des squelettes de Sphaerellaires (Radiolaires) en voie de sédimentation dans les vases de la Méditerranée. Incidences sur la systématique de ces formes. C. R. Acad. Sci. Ser. 2, 274:2759-2762.

1974. Les Radiolaires des boues superficielles de la Méditerranée (The Radiolaria of the surficial sediments of the Mediterranean Sea). Bull. Mus. Natl. Hist. Nat. Sci. Terr., 249:217-288.

1977. La silice biogène dans les sédiments néogènes et quaternaires de l'océan Indien austral. Bull. Soc. Geol. Fr., 19:1021-1032.

Dumitrica, P., 1973a. Cretaceous and Quaternary Radiolaria in deep-sea sediment from the northwest Atlantic Ocean and Mediterranean Sea. In Ryan, W.B.F., Hsü, K.J., et al., Init. Repts. DSDP, 13: Washington (U.S. Govt. Printing Office), 829-901.

, 1973b. Miocene and Quaternary silicoflagellates in sediments from the Mediterranean Sea. In Ryan, W.B.F., Hsü, K.J., et al., Init. Repts. DSDP, 13: Washington (U.S. Govt. Printing Office), 902-933.

,1973c. Paleocene, late Oligocene and post-Oligocene silicoflagellates in southwestern Pacific sediments cored on DSDP Leg 21. In Burns, R.E., Andrews, J.E., et al., Init. Repts. DSDP, 21: Washington (U.S. Govt. Printing Office), 837-883.

Dworetzky, B.A., and Morley, J.J., 1987. Vertical distribution of radiolaria in the eastern equatorial Atlantic: analysis of a multiple series of closelyspaced plankton tows. Mar. Micropaleontol., 12:1-19.

Ehrenberg, C.G., 1837. [Eine briefliche Nachricht des Hrn. Agassiz in Neuchatel] über den ebenfalls aus mikroskopischen Kiesel-Organismen gebildeten Polirschiefer von Oran in Africa. Ber. Bekannt. Verh. K. Preuss. Akad. Wiss. Berlin 1837: 59-61.

1839. Über die Bildung der Kreidefelsen und des Kreidemergels durch unsichtbare Organismen. K. Akad. Wiss. Berlin, Abh. 1838 (1840, separate 1839): 59-148.

- 1843. Verbreitung und Einfluß des mikroskopischen Lebens in Süd- und Nord-Amerika. Abh. Preuss. Akad. Wiss. Berlin Physik;Math. Kl., 1841:291-446.

, 1847. Uber die mikroskopischen kieselschaligen Polycystinen als machtige Gebirgmasse von Barbados und uber das Verhaltniss der aus mehr als 300 neuen Arten bestehenden ganz eigenthumlichen Formengruppe jener Felsmasse zu den lebenden Thieren und zur Kreidebildung. Eine neue Anregung zur Erforschung des Erdlebens. K. Preuss. Akad. Wiss. Berlin 1847: 40-60.

1858. Kurze Characteristik der 9 neuen Genera und der 105 neuen Species des ägäischen Meeres und des Tiefgrundes des MittelMeeres. $K$. Preuss. Akad. Wiss. Berlin 1858: 10-40.

Emeis, K.-C., and Shipboard Scientific Party, 1996. Paleoceanography and sapropel introduction. In Emeis, K.-C., Robertson, A.H.F., Richter, C., et al., Proc. ODP, Init. Repts., 160: College Station, TX (Ocean Drilling Program), 21-28. 
Fontugne, M.R., and Calvert, S.E., 1992. Late Pleistocene variability of the carbon isotopic composition of organic matter in the eastern Mediterranean: monitor of changes in carbon sources and atmospheric $\mathrm{CO}_{2}$ levels. Paleoceanography, 7:1-20.

Frenguelli, J., 1935. Variaciones de Dictyocha fibula en el Golfo de San Matias (Patagonia septentrional). An. Mus. Argentino Cienc. Nat. "Bernardino Rivadavia," 38:265-281.

, 1951. Silicoflagelados del Trípoli de Majillones (Chile). Physis, 20:272-284.

Frydas, D., 1986. Silicoflagellate associations, biostratigraphy and palaeoenvironment analysis of the Pliocene from section Aitania (Crete, Greece). Documenta naturae, 28:1-8.

1990. Stratigraphie des diatomites du Plaisancien de la Crete centrale (Grece) à l' aide des Silicoflagellés et des nannofossiles calcaires. Rev. Micropalaeontol., 33:93-114.

, 1994. Bericht uber ein neues Silicoflagellaten-Vorkommen aus dem Piacenzium von Kreta, Griechenland. Berliner Geowiss. Abh., E13:483-493.

_, 1996. Silicoflagellate stratigraphy for Neogene to Quaternary marine sediments in Greece. Newsl. Stratigr., 33:99-116.

Goll, R.M., 1979. The Neogene evolution of Zygocircus, Neosemantis and Callimitra: their bearing on nassellarian classification. A revision of the Plagiacanthoidea. Micropaleontology, 25:365-396.

Goll, R.M., and Bjørklund, K.R., 1989. A new radiolarian biostratigraphy for the Neogene of the Norwegian Sea: ODP Leg 104. In Eldholm, O., Thiede, J., Taylor, E., et al., Proc. ODP, Sci. Results, 104: College Station, TX (Ocean Drilling Program), 697-737.

Haeckel, E., 1860a. Über neue, lebende Radiolarien des Mittelmeeres. $K$. Preuss. Akad. Wiss. Berlin 1860: 794-817.

— 1860b. Fernere Abbildungen und Diagnosen neuer Gattungen und Arten von lebenden Radiolarien des Mittelmeeres. K. Preuss. Akad. Wiss. Berlin 1860: 835-845.

, 1862. Die Radiolarien (Rhizopoda Radiolaria): Berlin (Reimer).

, 1881. Entwurf eines Radiolarien-Systems auf Grund von Studien der Challenger-Radiolarien. Jena Z. Med. Naturwiss., 15:418-472.

, 1887. Report on the Radiolaria collected by H.M.S. Challenger during the years 1873-1876. Rept. Voyage Challenger, Zool., 18:1-1803.

Howell, M.W., and Thunell, R.C., 1992. Organic carbon accumulation in Bannock Basin: evaluating the role of productivity in the formation of Eastern Mediterranean sapropels. Mar. Geol., 103:461-471.

Kling, S.A., 1979. Vertical distribution of polycystine radiolarians in the central North Pacific. Mar. Micropaleontol., 4:295-318.

Krom, M.D., Brenner, S., Israilov, L., and Krumgalz, B., 1991a. Dissolved nutrients, preformed nutrients and calculated elemental ratios in the South-East Mediterranean Sea. Oceanol. Acta, 14:189-194.

Krom, M.D., Kress, N., Brenner, S., and Gordon, L.I., 1991b. Phosphorus limitation of primary productivity in the eastern Mediterranean Sea. Limnol. Oceanogr., 36:424-432.

Lemmermann, E., 1901. Silicoflagellatae. Ergebnisse einer Reise nach dem Pacific. H. Schauinsland, 1896-97. Ber. Dtsch. Bot. Ges., 19:247-271.

Ling, H.Y., 1970. Silicoflagellates from central North Pacific core sediments. Bull. Am. Paleontol., 58:85-129.

, 1972. Upper Cretaceous and Cenozoic silicoflagellates and ebridians. Bull Am. Paleontol., 62:135-229.

Lipps, J.H., 1970. Ecology and evolution of Silicoflagellates. In Yochelson, E.L. (Ed.), Proc. North Am. Paleontol. Conv., 2:965-993.

Locker, S., 1974. Revision der Silicoflagellaten aus der Mikrogeologischen Sammlung von C.G. Ehrenberg. Eclogae Geol. Helv., 67:631-646.

, 1995. Silicoflagellates, ebridians, and actiniscidians from Pliocene and Quatemary sediments off southern Chile, Ocean Drilling Project Leg 141. In Lewis, S.D., Behrmann, J.H., Musgrave, R.J., and Cande, S.C. (Eds.), Proc. ODP, Sci. Results, 141: College Station, TX (Ocean Drilling Program), 223-233.

Locker, S., and Martini, E., 1986. Silicoflagellates and some sponge spicules from the southwest Pacific, DSDP Leg 90. In Kennett, J.P., von der Borch, C.C., et al., Init. Repts. DSDP, 90: Washington (U.S. Govt. Printing Office), 887-924.

Loeblich, A., III, Loeblich, L.A., Tappan, H., and Loeblich, A.R., Jr., 1968. Annotated index of fossil and recent silicoflagellates and ebridians with descriptions and illustrations of validly proposed taxa. Mem.-Geol. Soc. Am., 106:1-319.

Ludwig, J.R., and Volcani, B.E., 1986. A molecular biology approach to understanding silicon metabolism in diatoms. In Leadbeater, B.S.C., and
Riding, R. (Eds.), Biomineralization in Lower Plants and Animals. Syst. Assoc. Spec. Vol., 30:315-326.

Martini, E., 1971. Standard Tertiary and Quaternary calcareous nannoplankton zonation. In Farinacci, A. (Ed.), Proc. 2nd Int. Conf. Planktonic Microfossils Roma: Rome (Ed. Tecnosci.), 2:739-785.

, 1976. Neogene and Quaternary silicoflagellates from the Central Pacific Ocean (DSDP Leg 33). In Schlanger, S.O., Jackson, E.D., et al., Init. Repts. DSDP, 33: Washington (U.S. Govt. Printing Office), 439449.

1990. Tertiary and Quaternary silicoflagellates, actiniscidians, and ebridians from the eastern Pacific off Peru (Leg 112). In Suess, E., von Huene, R., et al., Proc. ODP, Sci. Results, 112: College Station, TX (Ocean Drilling Program), 157-173.

McCartney, K., Churchill, S., and Woestendiek, L., 1995. Silicoflagellates and Ebridians from Leg 138, Eastern Equatorial Pacific. In Pisias, N.G., Mayer, L.A., Janecek, T.R., Palmer-Julson, A., and van Andel, T.H. (Eds.), Proc. ODP, Sci. Results, 138: College Station, TX (Ocean Drilling Program), 129-162.

McMillen, K.J., and Casey, R.E., 1978. Distribution of living polycystine radiolarians in the Gulf of Mexico and Caribbean Sea, and composition with sedimentary record. Mar. Micropaleontol., 3:121-145.

Müller, J., 1858. Uber die Thalassicollen, Polycystinen und Acanthometren des Mittelmeeres. Abh. Kgl. Akad. Wiss. Berlin 1858: 1-62.

Nigrini, C., and Moore, T.C., 1979. A Guide to Modern Radiolaria. Spec. Publ. Cushman Found. Foraminiferal Res., 16.

Okada, H., and Bukry, D., 1980. Supplementary modification and introduction of code numbers to the low-latitude coccolith biostratigraphic zonation (Bukry, 1973; 1975). Mar. Micropaleontol., 5:321-325.

Ormiston, A.R., 1993. The association of radiolarians with hydrocarbon source rocks. Micropaleontology, Spec. Publ., 6:9-16.

Pedersen, T.F., and Calvert, S.E., 1990. Anoxia vs. productivity: what controls the formation of organic-carbon-rich sediments and sedimentary rocks? AAPG Bull., 74:454-466.

Perch-Nielsen, K., 1975. Late Cretaceous to Pleistocene silicoflagellates from the southern Southwest Pacific, DSDP, Leg 29. In Kennett, J.P., Houtz, R.E., et al., Init. Repts. DSDP, 29: Washington (U.S. Govt. Printing Office), 677-721.

Perch-Nielsen, K., 1985. Silicoflagellates. In Bolli, H.M., Saunders, J.B., and Perch-Nielsen, K. (Eds.), Plankton Stratigraphy: Cambridge (Cambridge Univ. Press), 811-846.

Peters, W., 1860. Kurzen Auszug aus einer Abhandlung des Hrn. Dr. Ernst Haeckel über neue, ledende Radiolarien des Mittelmeeres und legte die dazu gehörigen Abbildungen vor. Mber. Verh. K. Preuss. Akad. Wiss. Berlin, 794-817.

Poelchau, H.S., 1976. Distribution of Holocene silicoflagellates in North Pacific sediments. Micropaleontology, 22:164-193.

Qiu, L., Williams, D.F., Gvorzdkov, A., Karabanov, E., and Shimaraeva, M., 1993. Biogenic silica accumulation and paleoproductivity in the northern basin of Lake Baikal during the Holocene. Geology, 21:25-28.

Riedel, W.R., 1967a. Some new families of Radiolaria. Proc. Geol. Soc. London, 1640:148-149.

, 1967b. Subclass Radiolaria. In Harland, W.B., Holland, C.H., House, M.R., Hughes, N.F., Reynolds, A.B., Rudwick, M.J.S., Satterthwaite, G.E., Tarlo, L.B.H., and Willey, E.C. (Eds.), The Fossil Record. Geol. Soc. London, 291-298.

Riedel, W.R., Sanfilippo, A., and Cita, M.B., 1974. Radiolarians from the stratotype Zanclean (lower Pliocene, Sicily). Riv. Ital. Paleontol. Stratigr., 80:699-734.

Riedel, W.R., Westberg-Smith, M.J., and Budai, A., 1985. Late Neogene Radiolaria and Mediterranean paleoenvironments. In Stanley, D.J., and Wezel, F.-C. (Eds.), Geological Evolution of the Mediterranean Basin: Berlin (Springer-Verlag), 487-523.

Rio, D., Raffi, I., and Villa, G., 1990. Pliocene-Pleistocene calcareous nannofossil distribution patterns in the Western Mediterranean. In Kastens, K.A., Mascle, J., et al., Proc. ODP, Sci. Results, 107: College Station, TX (Ocean Drilling Program), 513-533.

Robertson, A., and Ocean Drilling Program Leg 160 Scientific Party, 1996. Mud volcanism on the Mediterranean Ridge: Initial results of Ocean Drilling Program Leg 160. Geology, 24:239-242.

Rohling, E.J., 1994. Review and new aspects concerning the formation of eastern Mediterranean sapropels. Mar. Geol., 122:1-28.

Rohling, E.J., and Gieskes, W.W.C., 1989. Late Quaternary changes in Mediterranean intermediate water density and formation rate. Paleoceanography, 4:531-545. 
Rossignol-Strick, M., 1983. African monsoons: an immediate climate response to orbital insolation. Nature, 304:46-49.

Sancetta, C., 1994. Mediterranean sapropels: Seasonal stratification yields high production and carbon flux. Paleoceanography, 9:195-196.

Sanfilippo, A., 1971. Neogene radiolarians of the Mediterranean and western Pacific. In Farinacci, A., (Ed.), Proc. 2nd Planktonic Conf., Roma 1970: Rome (Edizioni Tecnoscienza), 1121-1127.

Sanfilippo, A., Burckle, L.H., Martini, E., and Riedel, W.R., 1973. Radiolarians, diatoms, silicoflagellates and calcareous nannofossils in the Mediterranean Neogene. Micropaleontology, 19:209-234.

Sanfilippo, A., Caulet, J.-P., and Riedel, W.R., 1978. Radiolaria from Mediterranean sediments, DSDP Leg 42A. In Hsü, K.J., Montadert, L., et al., Init. Repts. DSDP, 42 (Pt.1): Washington (U.S. Govt. Printing Office), $753-760$.

Sanfilippo, A., and Riedel, W.R., 1975. Late Tertiary radiolarians from Crete. Proc. 6th Congr. Regional Committee on Mediterranean Neogene Stratigr., Bratislava 1975, 61-74.

Sanfilippo, A., and Riedel, W.R., 1980. A revised generic and suprageneric classification of the Artiscins (Radiolaria). J. Paleontol., 54:1008-1011.

Schrader, H., 1971. Fecal pellets: their role in the sedimentation of pelagic diatoms. Science, 174:55-57.

Schrader, H., and Matherne, A., 1981. Sapropel formation in the eastern Mediterranean Sea: Evidence from preserved opal assemblages. Micropaleontology, 27:191-203.

Shipboard Scientific Party, 1996a. Site 969. In Emeis, K.-C., Robertson, A.H.F., Richter, C., et al., Proc. ODP, Init. Repts., 160: College Station, TX (Ocean Drilling Program), 335-375.
, 1996b. Site 971. In Emeis, K.-C., Robertson, A.H.F., Richter, C., et al., Proc. ODP, Init. Repts., 160: College Station, TX (Ocean Drilling Program), 415-450.

Stradner, H., and Bachmann, A., 1978. Late Pliocene and Early Pleistocene silicoflagellates and ebridians from DSDP site 378 in the Aegean basin, North of Crete. In Hsü, K., Montadert, L., et al., Init. Repts. DSDP, 42 (Pt. 1): Washington (U.S. Govt. Printing Office), 805-815.

Takahashi, K., and Honjo, S., 1981. Vertical flux of radiolaria: A taxon-quantitative sediment trap study from the western tropical Atlantic. Micropaleontology, 27:140-190.

Ten Haven, H.L., De Lange, G.J., and McDuff, R.E., 1987. Interstitial water studies of late Quaternary Eastern Mediterranean sediments with emphasis on early diagenetic reactions and evaporitic salt influences. Mar. Geol., 75:119-136.

Van de Paverd, P.J., 1995. Recent polycystine Radiolaria from the Snellius-II expedition [Ph.D. dissert.], Amsterdam.

Date of initial receipt: 10 January 1997

Date of acceptance: 19 June 1997

Ms 160SR-007 


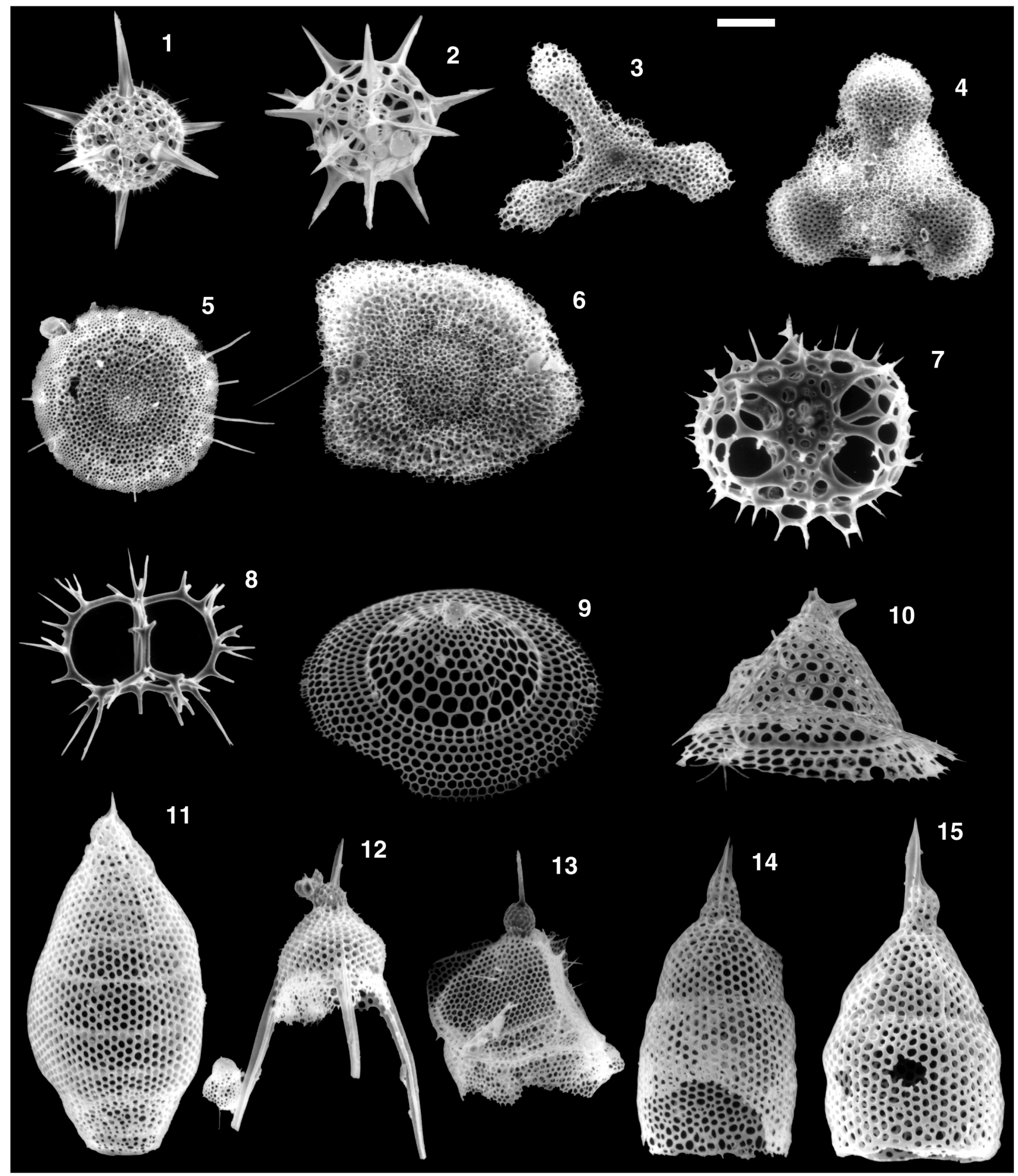

Plate 1. Scanning electron micrographs of radiolarians from Sample 160-971B-2H-CC. Numbers in $\mu \mathrm{m}$ in all figures refer to the same scale bar (upper right). 1. Hexacontium group, $67 \mu \mathrm{m}$; 2. Other actinommids, $48 \mu \mathrm{m}$; 3-4. Dictyocoryne group: (3) $72 \mu \mathrm{m}$; (4) D. truncatum, $75 \mu \mathrm{m} ; 5$. Porodiscus group, 97 $\mu \mathrm{m} ;$ 6. Spongaster sp., $67 \mu \mathrm{m}$; 7. Pyloniid group, $45 \mu \mathrm{m} ; 8$. Acanthodesmia vinculata, $76 \mu \mathrm{m} ; 9-10$. Eucecryphalus group: (9) $45 \mu \mathrm{m}$, (10) $43 \mu \mathrm{m} ; 11$. Eucyrtidium acuminatum, $32 \mu \mathrm{m}$; 12. Pterocanium group, $53 \mu \mathrm{m}$; 13. Other theoperids: Lipmanella bombus, $43 \mu \mathrm{m} ; \mathbf{1 4 - 1 5}$. Three-segmented pterocorythid: (14) $43 \mu \mathrm{m},(15) 44 \mu \mathrm{m}$. 

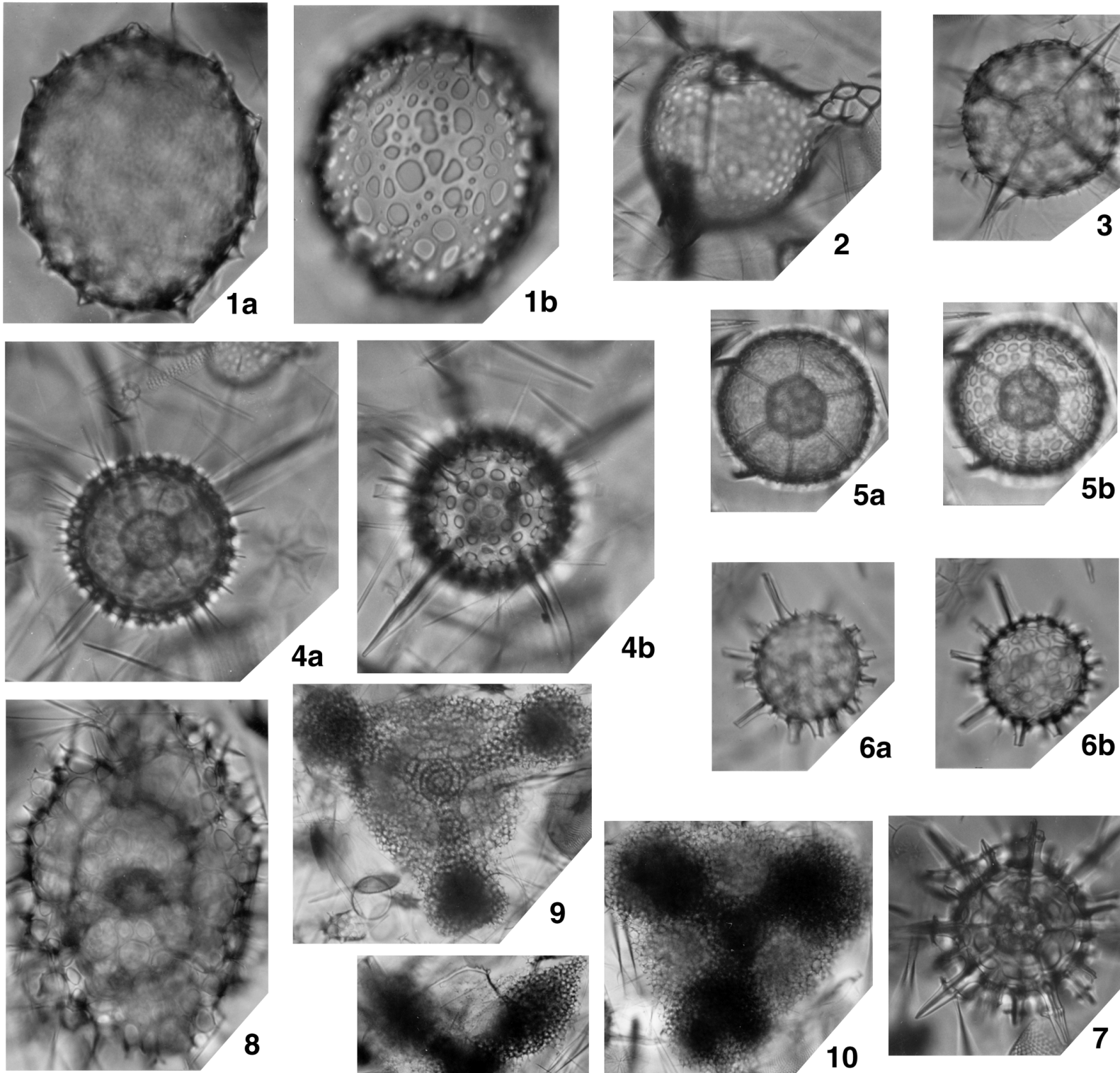

$4 b$
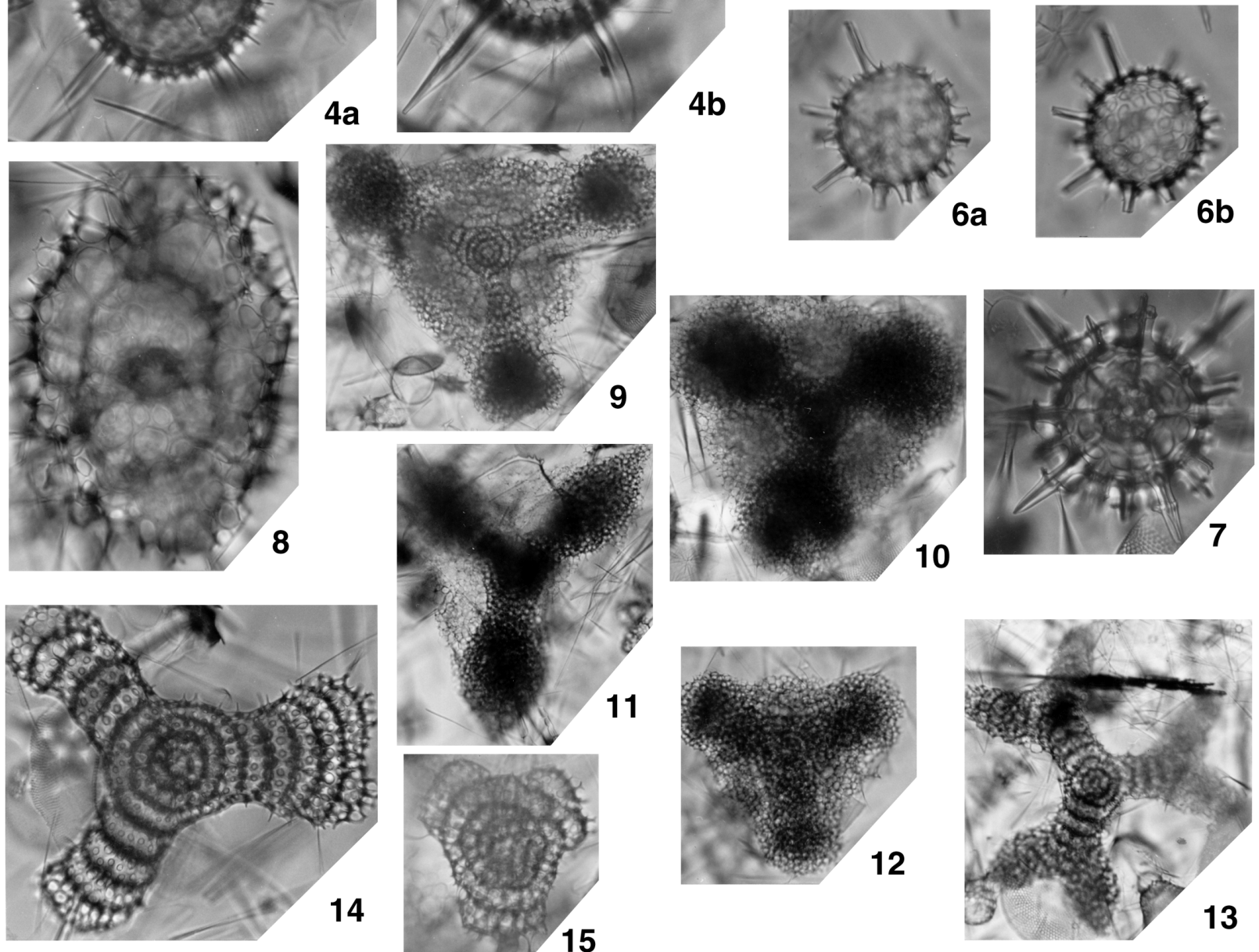

Plate 2. Radiolarians from Sample 160-971B-2H-CC. Magnification 240× for all figures apart from 9 to 13 (120×). 1-2. Collosphaerid group: (1) Acrosphaera spinosa group?; (2) Solenosphaera chierchiae; 3-4. Hexacontium group; 5. Thecosphaera inermis; 6-7. Other actinommids; 8. Didymocyrtis sp.; 9-12. Dictyocoryne group: (9) D. profunda; (10) D. truncatum; 13-15. Hymeniastrum group: (13) H. regulare; (14) H. virchowii. 

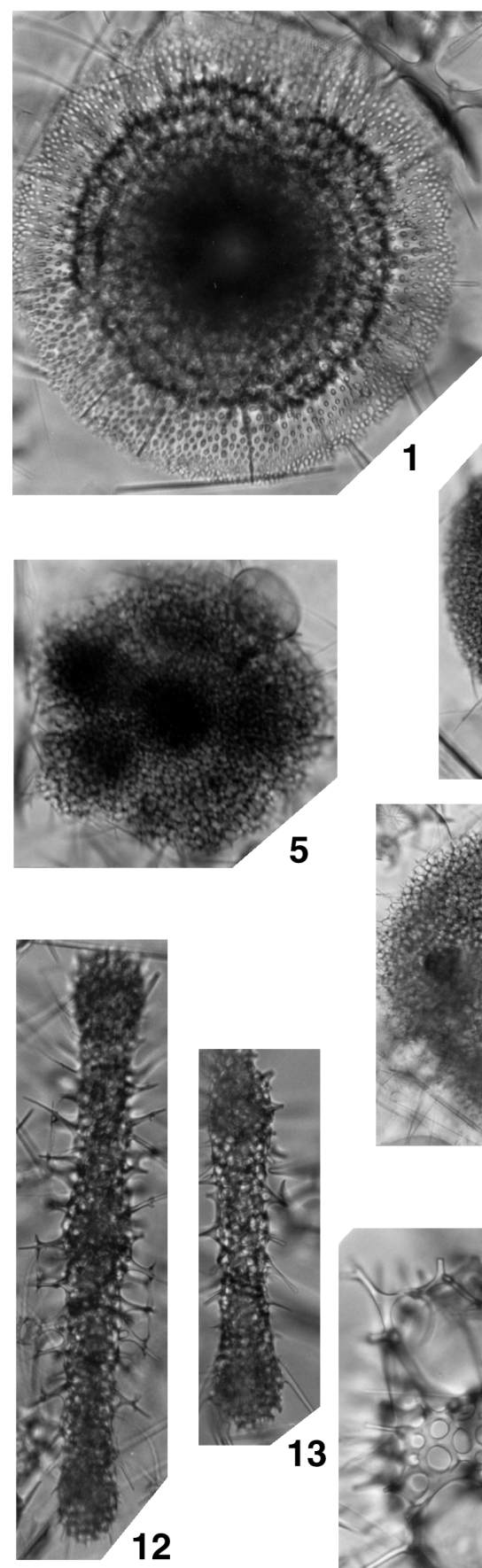

13

12
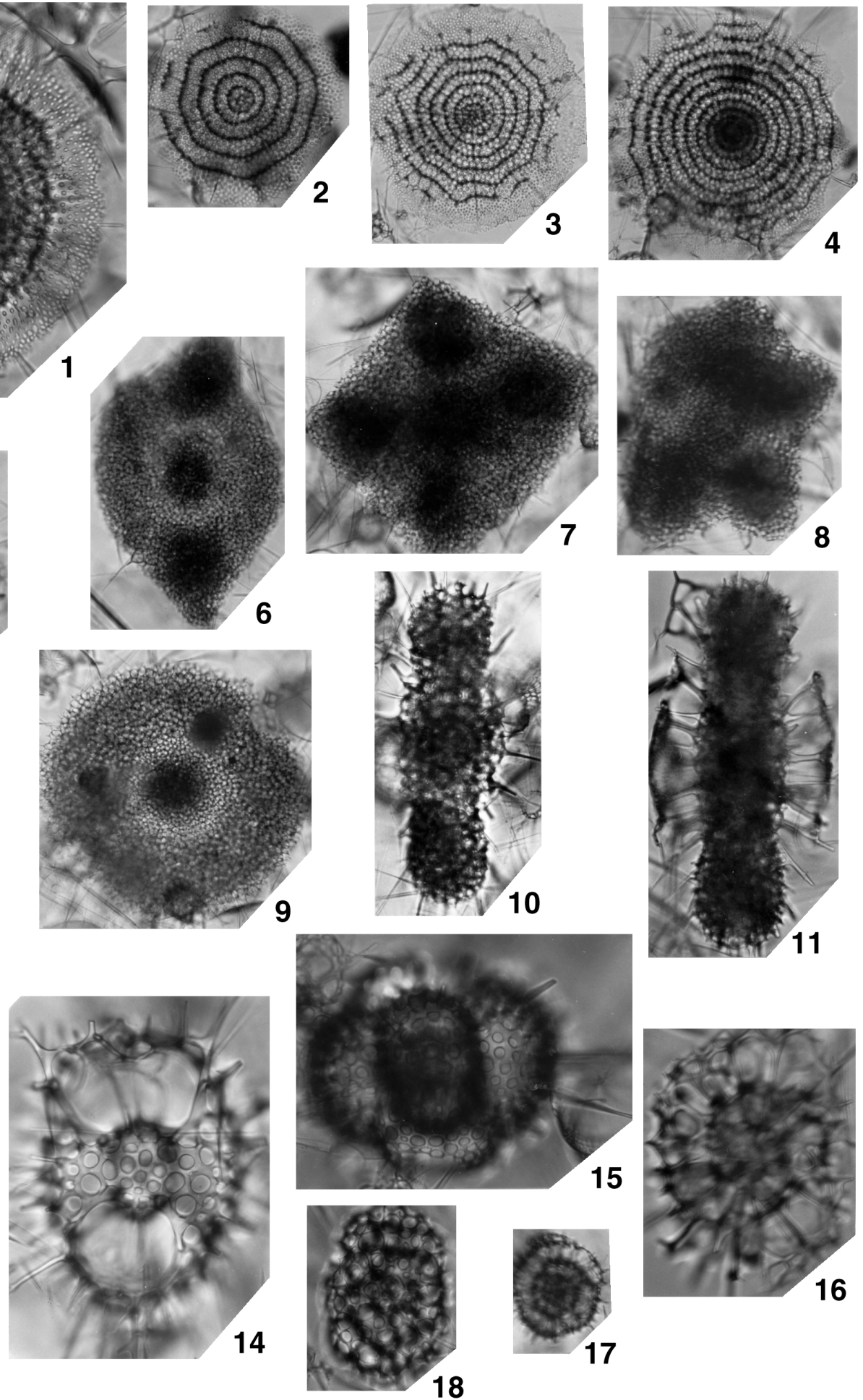

Plate 3. Radiolarians from Sample 160-971B-2H-CC. Magnification 120× for Figures 2-9, 17 and 240× for Figures 1, 10-16, 18. 1-4. Porodiscus group: (1) Porodiscus group A; (2-3) Porodiscus group B; 5-8. Spongaster spp.; 9. Spongodiscus group; 10-13. Spongocore spp.: (10-11) Spongocore puella; 14. Pyloniid group; 15. Tholoniid group: Amphitholus metallasson; 16-18. Pylospira group, (16) P. octopyle. 

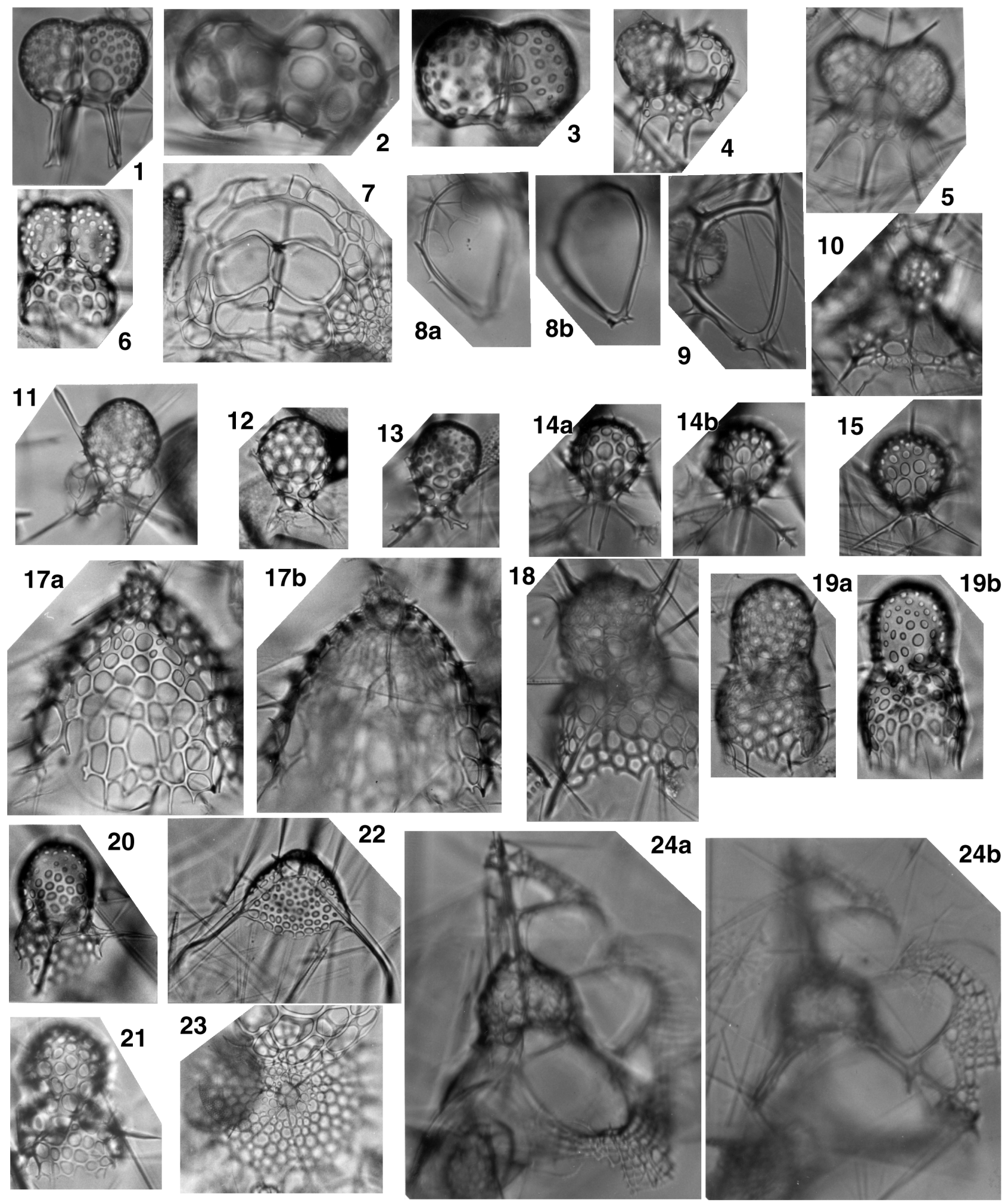

Plate 4. Radiolarians from Sample 160-971B-2H-CC. Magnification 300× for all figures apart from 7 and 22 (150×). 1. Ceratospyris group A; 2-3. Ceratospyris group B; 4-5. Ceratospyris group C; 6. Desmospyris group; 7. Nephrospyris renilla; 8-9. Zygocircus group: (8) Z. productus productus; (9) Z. productus piscicaudatus; 10. Arachnocorys circumtexta; 11-15. Arachnocorallium group; 17. Ceratocyrtis group; 18-21. Lophophaena group; 22. Pseudodictyophimus group; 23. Sethophormin group; 24. Other plagoniids: Clathrocorys teuscheri. Note that there is not a Figure 16. 

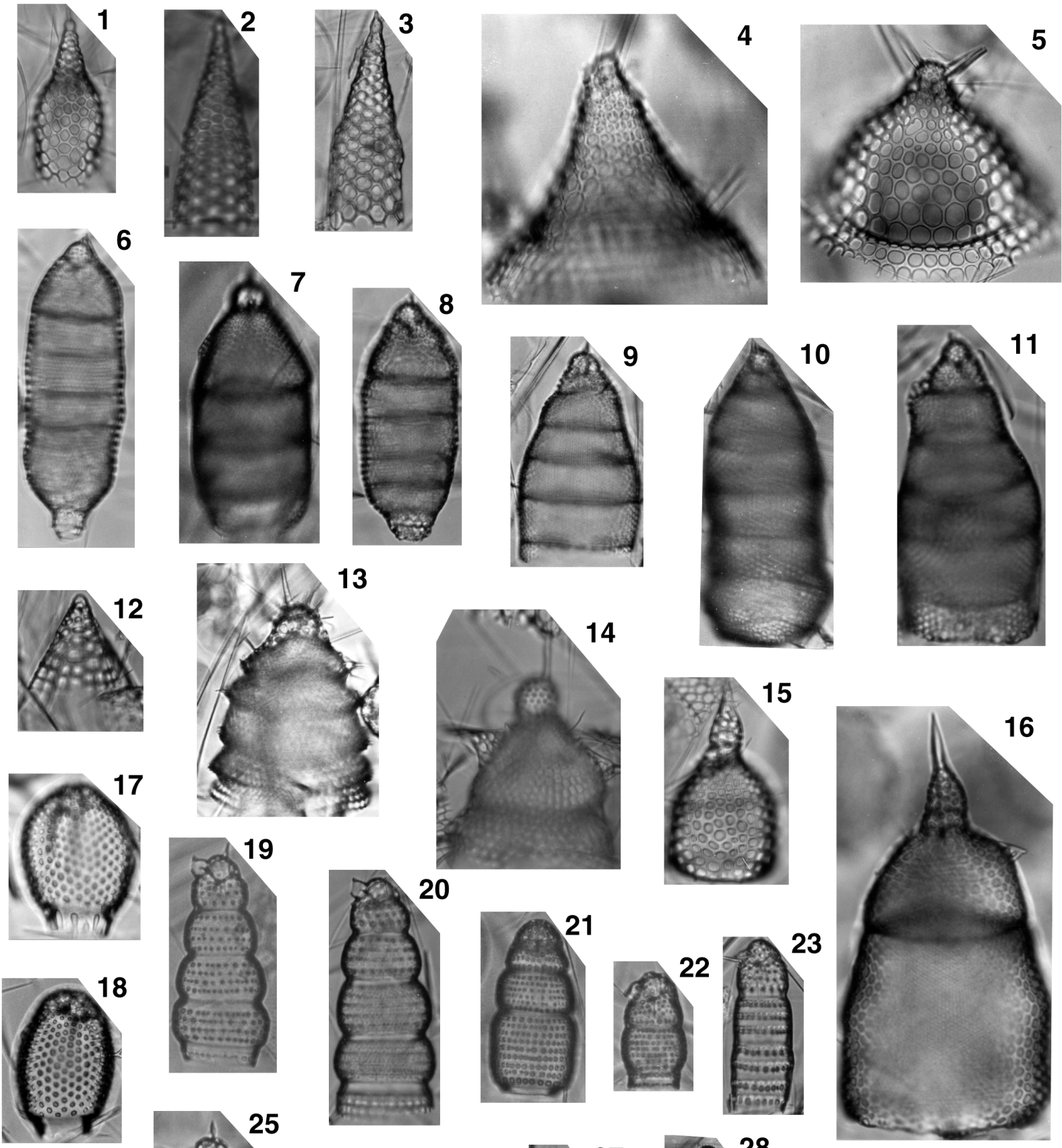

\section{5}
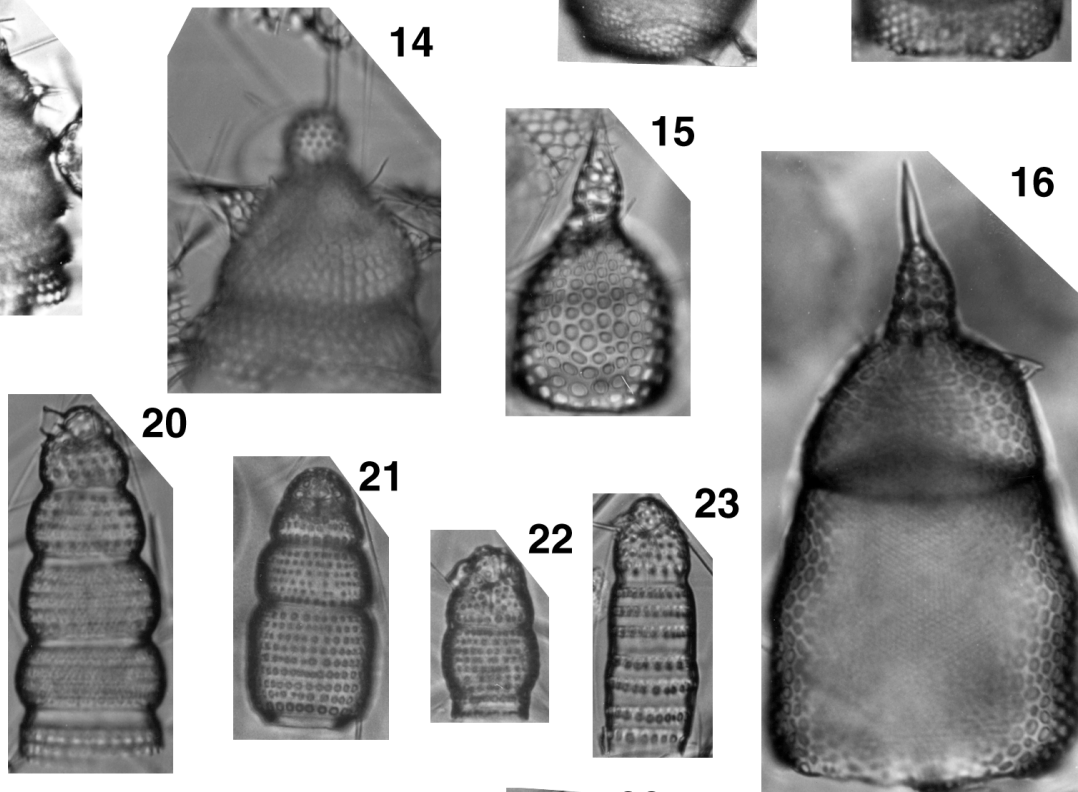

24
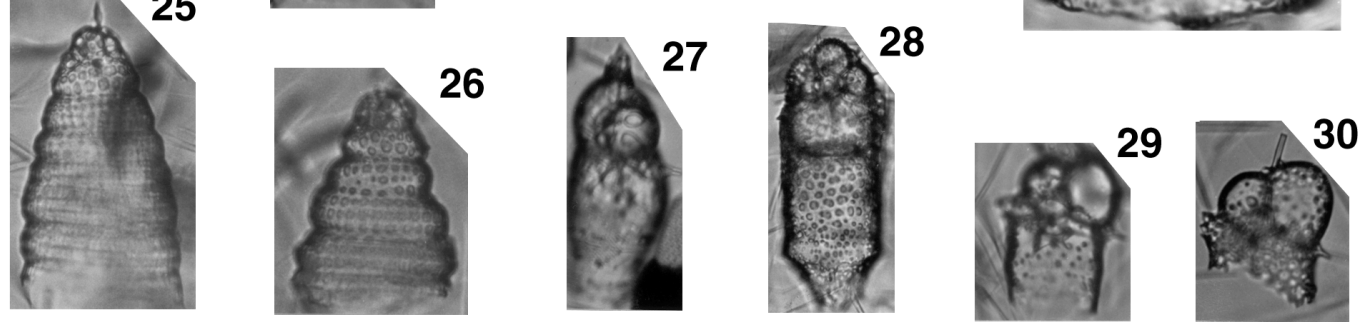

Plate 5. Radiolarians from Sample 160-971B-2H-CC. Magnification 240× for all figures. 1-3. Cornutella spp.: (2-3) Cornutella profunda; 4-5. Eucecryphalus group; 6-8. Eucyrtidium punctatum group; 9-11. Other Eucyrtidium: (9) E. acuminatum; 12. Litharachnium group; 1314. Other theoperids: (13) Lithostrobus hexagonalis; (14) Lipmanella dictyoceras; 15. Anthocyrtidium group; 16. Three-segmented pterocorythid. 17-18. Carpocanistrum group A; 19-20. Botryostrobus group; 21-22. Phormostichoartus group; 23-24. Siphocampe group; 25-26. Spirocyrtis group; 27-30. Cannobotryid group. 

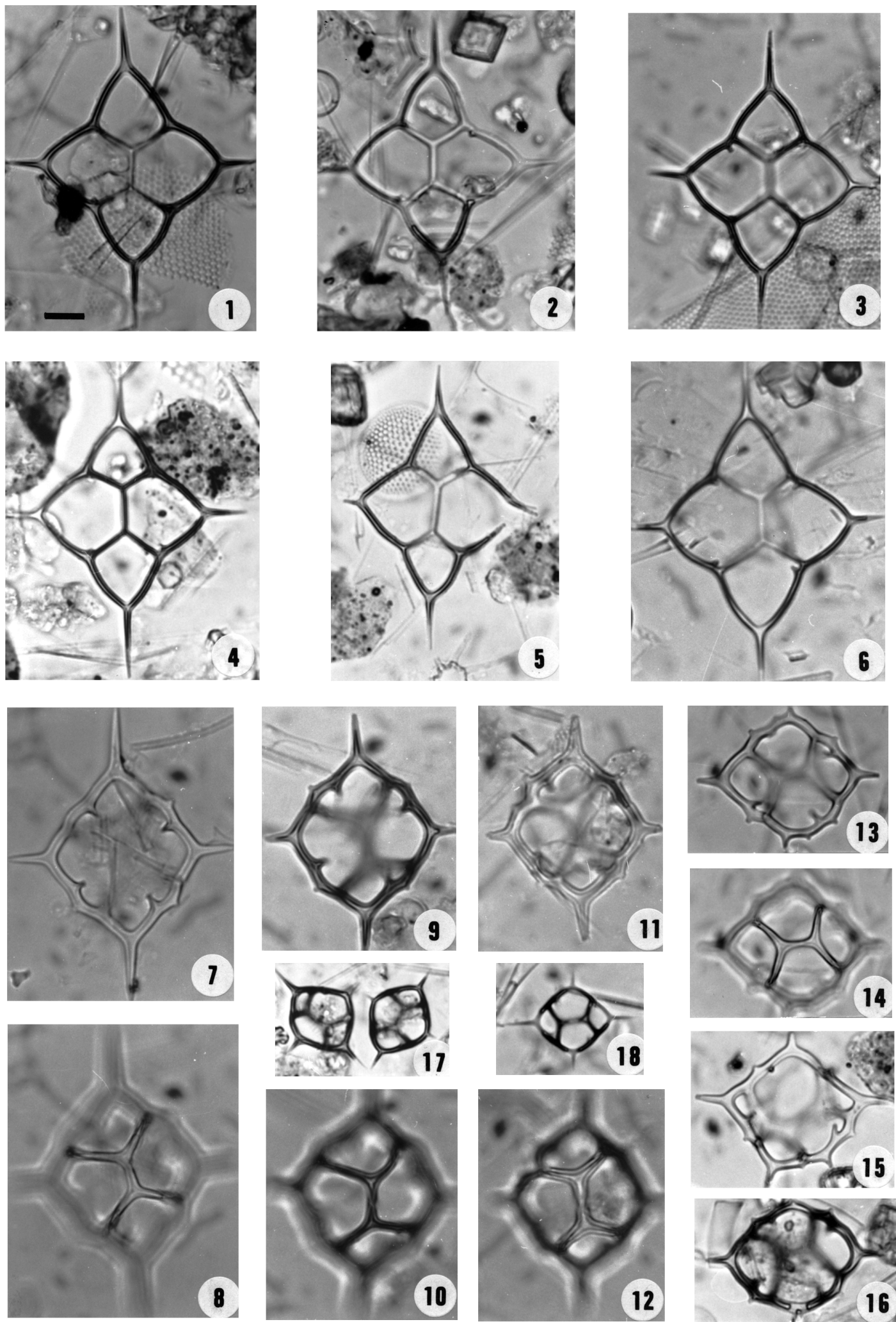

Plate 6. Silicoflagellates from Sample 160-971B-2H-CC. Magnification 600× for all figures; scale bar equals $10 \mu \mathrm{m}$. 1-6. Dictyocha calida ampliata: $(1-4,6)$ normal forms; (5) irregular form; 7-16. D. aculeata aculeata: (7-12) large forms; (1316) small forms; $(7,9,11,13,15,16)$ focused on basal ring; $(8,10,12,14)$ focused on apical bar; (15) pentagonal specimen; 17-18. D. messanensis, small rhomboidal forms. 

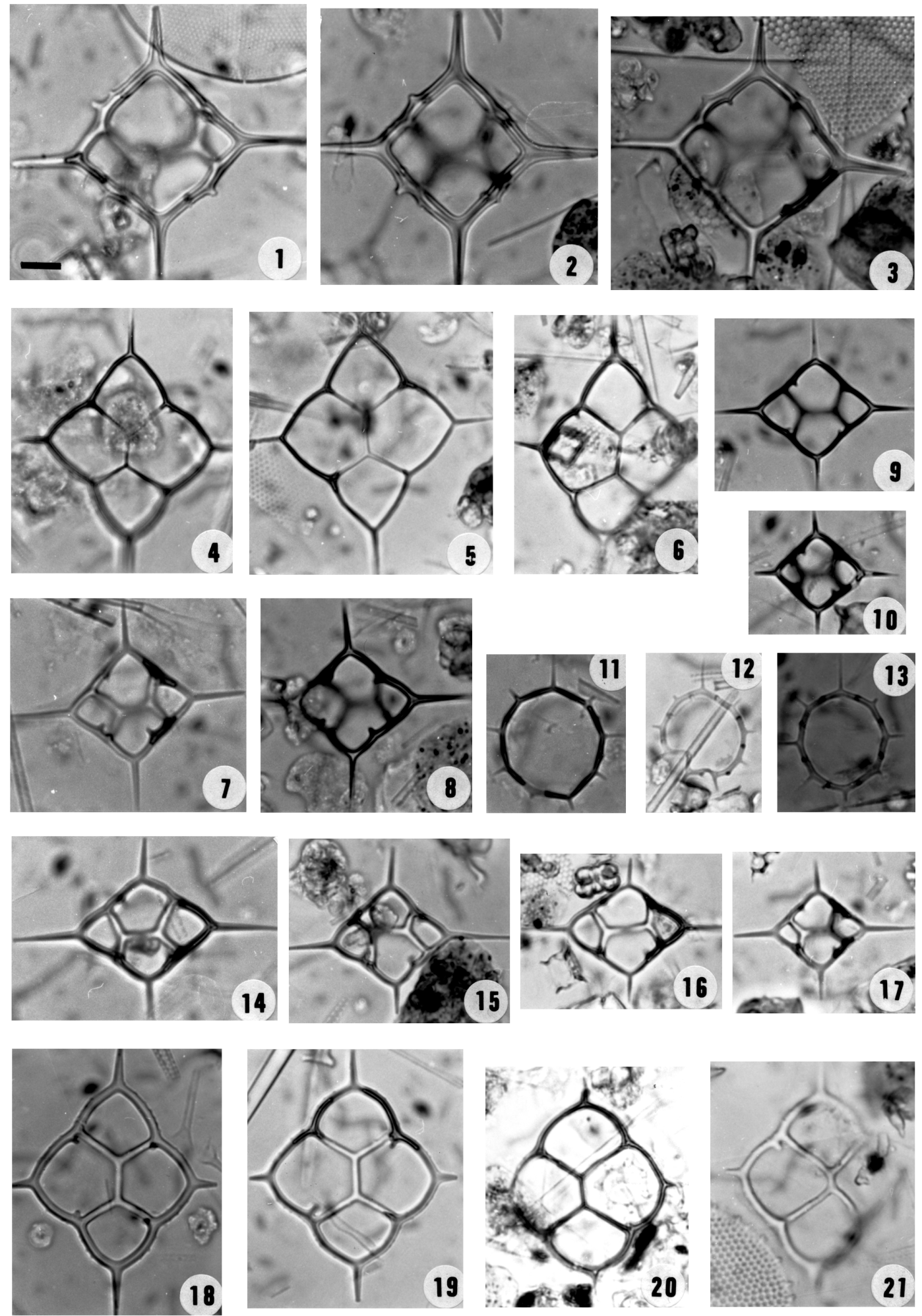

Plate 7. Silicoflagellates from Sample 160-971B-2H-CC. Magnification $600 \times$ for all figures; scale bar equals $10 \mu \mathrm{m} .1-3$, 7-10. D. stapedia aspinosa: (1-3) large forms, focused on basal ring; (7-10) small forms, focused on basal ring; 4-6. D. delicata: 4-6 fibuloid forms with small apical bar. 11-12. Distephanus octogonus, nine-rayed forms; 13. D. octogonus, eight-rayed form. 14-17. Dictyocha stapedia stapedia, rhomboidal forms with long radial spines in the major axis. 18-19. D. fibula; 20-21. D. perlaevis perlaevis; asperoid forms. 